TRANSACTIONS OF THE

AMERICAN MATHEMATICAL SOCIETY

Volume 362, Number 5, May 2010, Pages 2301-2337

S 0002-9947(09)05069-7

Article electronically published on December 8, 2009

\title{
TRACES OF HEAT OPERATORS ON RIEMANNIAN FOLIATIONS
}

\author{
KEN RICHARDSON
}

\begin{abstract}
We consider the basic heat operator on functions on a Riemannian foliation of a compact, Riemannian manifold, and we show that the trace $K_{B}(t)$ of this operator has a particular asymptotic expansion as $t \rightarrow 0$. The coefficients of $t^{\alpha}$ and of $t^{\alpha}(\log t)^{\beta}$ in this expansion are obtainable from local transverse geometric invariants - functions computable by analyzing the manifold in an arbitrarily small neighborhood of a leaf closure. Using this expansion, we prove some results about the spectrum of the basic Laplacian, such as the analogue of Weyl's asymptotic formula. Also, we explicitly calculate the first two nontrivial coefficients of the expansion for special cases such as codimension two foliations and foliations with regular closure.
\end{abstract}

\section{INTRODUCTION}

Let $M$ be an $n$-dimensional, compact, connected, oriented Riemannian manifold without boundary. The heat kernel is the fundamental solution to the associated heat equation. That is, it is the unique function $K:(0, \infty) \times M \times M$ that satisfies

$$
\begin{aligned}
\left(\frac{\partial}{\partial t}+\Delta_{x}\right) K(t, x, y) & =0 \quad \text { and } \\
\lim _{t \rightarrow 0^{+}} \int_{M} K(t, x, y) f(y) \operatorname{dvol}(y) & =f(x) \quad \text { for every continuous function } f .
\end{aligned}
$$

This function $K$ can be used to solve the heat equation $\left(\frac{\partial}{\partial t}+\Delta_{x}\right) g(t, x)=0$ for any initial temperature distribution $g(0, x)$. It is well known ([35]; see also [14, 49) that for any $x \in M$ and any positive integer $k$,

$$
K(t, x, x)=\frac{1}{(4 \pi t)^{n / 2}}\left(u_{0}(x)+u_{1}(x) t+\cdots+u_{k}(x) t^{k}+\mathcal{O}\left(t^{k+1}\right)\right) \text { as } t \rightarrow 0,
$$

where $u_{j}(x)$ are smooth functions on $M$ that depend only on geometric data at the point $x \in M$. In particular, $u_{0}(x)=1$ and $u_{1}(x)=\frac{S(x)}{6}$, where $S(x)$ is the scalar curvature of $M$ at $x$. Using the expansion above, it is possible to prove that the trace of the heat kernel has a similar asymptotic formula. Let $0=\lambda_{0}<\lambda_{1} \leq \lambda_{2} \leq \lambda_{3} \ldots$

Received by the editors October 8, 2007.

2010 Mathematics Subject Classification. Primary 53C12, 58J37, 58J35, 58J50.

Key words and phrases. Foliation, heat equation, asymptotics, basic, Laplacian.

The author's research at MSRI was supported in part by NSF grant DMS-9701755.

(C)2009 American Mathematical Society Reverts to public domain 28 years from publication 
be the eigenvalues of the Laplacian, counting multiplicities. Then

$$
\begin{aligned}
\operatorname{tr}\left(e^{-t \Delta}\right) & =\sum_{m \geq 0} e^{-t \lambda_{m}}=\int_{M} K(t, x, x) \operatorname{dvol}(x) \\
& =\frac{1}{(4 \pi t)^{n / 2}}\left(U_{0}+U_{1} t+\cdots+U_{k} t^{k}+\mathcal{O}\left(t^{k+1}\right)\right),
\end{aligned}
$$

where $U_{j}=\int_{M} u_{j}(x) \operatorname{dvol}(x)$, with $u_{j}(x)$ defined as above. In particular, $U_{0}=$ $\operatorname{Vol}(M)$. From formula (1.2), Karamata's theorem (see, for example, 24, pp. 418423]) implies the Weyl asymptotic formula ([51]; see also [14, p. 155]):

$$
\begin{aligned}
N(\lambda):=\quad \#\left\{\lambda_{m} \mid \lambda_{m} \leq\right. & \lambda\} \\
& \sim \quad \frac{\operatorname{Vol}(M)}{(4 \pi)^{n / 2} \Gamma\left(\frac{n}{2}+1\right)} \lambda^{n / 2}
\end{aligned}
$$

as $\lambda \rightarrow \infty$.

The heat kernel has also been studied more generally, such as in the case of manifolds with boundary or in the case of elliptic operators acting on sections of a vector bundle over the manifold. Many researchers have studied this expansion and its generalizations and have worked to compute the coefficient functions (see [35], [3], [33, 25]), because the heat kernel is not only used to compute heat flows but is also used in many areas of geometric and topological analysis. The asymptotic expansions above (and their generalizations) have been used to study the spectrum of the Laplacian (see [3, 4], 14], 33]), the determinant of the Laplacian (see [39], 46]), conformal classes of metrics (see [40]), analytic torsion (see [4], 15]), modular forms (see 23]), index theory (see [2], [49]), stochastic analysis (see [13], [32]), gauge theory/mathematical physics (see [22], [12, [6]), and so on.

Other researchers have studied generalizations of the heat kernel to orbit spaces of a group acting on a manifold. In [18, the author showed that if $M$ is a connected $n$-dimensional (not necessarily compact) Riemannian manifold and $\Gamma$ is a group acting isometrically, effectively, and properly discontinuously on $M$ with compact quotient $\bar{M}$, the induced heat operator $e^{-t \bar{\Delta}}$ on the space of functions on $\bar{M}$ (which is not necessarily a manifold) satisfies

$$
\operatorname{tr}\left(e^{-t \bar{\Delta}}\right)=\frac{1}{(4 \pi t)^{n / 2}}\left(\bar{U}_{0}+\bar{U}_{1} t+\ldots+\bar{U}_{k} t^{k}+\mathcal{O}\left(t^{k+1}\right)\right),
$$

where $n=\operatorname{dim}(M)$ and $\bar{U}_{0}=\operatorname{Vol}(\bar{M})$. This is equivalent to calculating the trace of the ordinary heat kernel on $M$ restricted to $\Gamma$-invariant functions. In 10, the researchers considered a compact, $n$-dimensional Riemannian manifold $M$ along with a compact group $G$ of isometries. Let $E_{\lambda}$ denote the complex eigenspace of $\Delta$ associated to the eigenvalue $\lambda$, and let $E_{\lambda}^{G}$ denote the subspace of $E_{\lambda}$ consisting of eigenfunctions invariant under the induced action of $G$. They show that the associated equivariant trace for $t>0$ is

$$
\begin{aligned}
L(t):= & \sum_{\lambda \geq 0} e^{-\lambda t} \operatorname{dim} E_{\lambda}^{G} \\
& \sim(4 \pi t)^{-m / 2}\left(a_{0}+\sum_{j>k \geq 0} a_{j k} t^{j / 2}(\log t)^{k}\right) \text { as } t \rightarrow 0,
\end{aligned}
$$


where $m=\operatorname{dim} M / G, K_{0}$ is less than or equal to the number of different dimensions of $G$-orbits in $M$, and $a_{0}=\operatorname{Vol}(M / G)$. The coefficients $a_{j k}$ depend only on the metrics on $M$ and $G$ and their derivatives on the subset $\{(g, x) \mid x g=x\} \subset G \times M$. The authors show in addition that under certain conditions, no logarithmic terms appear in the asymptotic expansion. Clearly, no logarithmic terms occur if all of the orbits have the same dimension. Also, if $G$ is connected of rank 1 and acts effectively on $M$, then no logarithmic terms appear. We remark that the results of [10] apply to more general situations. If a second order differential operator has the same principal symbol as the Laplacian, and if it is geometrically defined and thus commutes with the action of $G$, then the equivariant trace of the corresponding heat kernel satisfies (1.3). After writing this paper, the author was made aware of the recent work [19], where the authors compute the asymptotics of the heat kernel on orbifolds, related to the work in [18, [10], and to Theorem 3.5] in this paper.

In this paper, we consider a generalization of the trace of the heat kernel to Riemannian foliations, and we will observe asymptotic behavior similar to the results for group actions. Suppose that a compact, Riemannian manifold $M$ is equipped with a Riemannian foliation $\mathcal{F}$; that is, the distance from one leaf of $\mathcal{F}$ to another is locally constant. For simplicity, we assume that $M$ is connected and oriented and that the foliation is transversally oriented. In some sense this is a generalization of the work in 10 and 18, because the orbits of a group acting by isometries form an example of a Riemannian foliation if the orbits all have the same dimension. In [16, the authors explicitly calculated the heat kernel expansion in this specific case. Of course, the dimensions of orbits of arbitrary group actions on a manifold are typically not constant, and the leaf closures of a foliation are generally not orbits of a group action. In [48, we showed that many problems in the analysis of the transverse geometry of Riemannian foliations and that of group actions are equivalent problems.

A natural question to consider is the following: if we assume that the temperature is always constant along the leaves of $(M, \mathcal{F})$, how does heat flow on the manifold? To answer this question, we must restrict ourselves to the space of basic functions $C_{B}^{\infty}(M)$ (those that are constant on the leaves of the foliation) and more generally the space of basic forms $\Omega_{B}^{*}(M)$ (smooth forms $\omega$ such that given any vector $X$ tangent to the leaves, $i(X) \omega=0$ and $i(X) d \omega=0$, where $i(X)$ denotes the interior product with $X$ ). The exterior derivative $d$ maps basic forms to basic forms; let $d_{B}$ denote $d$ restricted to $\Omega_{B}^{*}(M)$. The relevant Laplacian on forms is the basic Laplacian $\Delta_{B}=d_{B} \delta_{B}+\delta_{B} d_{B}$, where $\delta_{B}$ is the adjoint of $d_{B}$ on $L^{2}\left(\Omega_{B}^{*}(M)\right)$. The basic heat kernel $K_{B}(t, x, y)$ on functions is a function on $(0, \infty) \times M \times M$ that is basic in each $M$ factor and that satisfies

$$
\begin{aligned}
\left(\frac{\partial}{\partial t}+\Delta_{B, x}\right) K_{B}(t, x, y) & =0 \\
\lim _{t \rightarrow 0^{+}} \int_{M} K_{B}(t, x, y) f(y) \operatorname{dvol}(y) & =f(x)
\end{aligned}
$$

for every continuous basic function $f$. The existence of the basic heat kernel allows us to answer the question posed at the beginning of this paragraph. The basic heat kernel on forms is defined in an analogous way. Many researchers have studied the analytic and geometric properties of the basic Laplacian and the basic heat kernel (see [1, 20], 21], 29, 37], 38, 42]). In 20, the author proved the existence of the basic heat kernel on functions. The existence of the basic heat kernel on forms 
was proved for the case where the mean curvature form of the foliation is basic in [37. The existence of the basic heat kernel was proved in general in [42, where the authors gave explicit formulas for the basic Laplacian and basic heat kernel in terms of the orthogonal projection from $L^{2}$-forms to $L^{2}$-basic forms and certain elliptic operators on the space of all forms on the manifold. A point of difficulty that often arises in this area of research is that the space of basic forms is not the set of all sections of any vector bundle, and therefore the usual theory of elliptic operators and heat kernels does not apply directly to $\Delta_{B}$ and $K_{B}$.

It is natural to try to prove the existence of asymptotic expansions of the form (1.1) and (1.2) for the basic heat kernel. We remark that the basic heat operator is of trace class, since the basic Laplacian is the restriction of an elliptic operator on the space of all functions (see lower bounds for eigenvalues in [42] and [31]). In [4], it was shown that an analogue of (1.1) exists for the basic heat kernel. As $t \rightarrow 0$, we have the following asymptotic expansion for any positive integer $k$ :

$$
K_{B}(t, x, x)=\frac{1}{(4 \pi t)^{q_{x} / 2}}\left(a_{0}(x)+a_{1}(x) t+\ldots+a_{k}(x) t^{k}+\mathcal{O}\left(t^{k+1}\right)\right)
$$

where $q_{x}$ is the codimension of the leaf closure containing $x$ and $a_{j}(x)$ are functions depending on the local transverse geometry and volume of the leaf closure containing $x$. The first two nontrivial coefficients were computed in [47] and are given in Theorem 3.15. In general, the power $q_{x}$ may vary, but its value is minimal and constant on an open, dense subset of $M$. One might guess that the asymptotics of the trace of the basic heat operator could be obtained by integrating the expansion (1.5), similar to obtaining (1.2) from (1.1). However, the functions $a_{j}(x)$ for $j \geq 1$ are not necessarily bounded or even integrable over the dense subset. Example 4.1 exhibits this precise behavior. Even if the coefficients $a_{j}(x)$ in (1.5) are integrable, it is not true in general that these functions can be integrated to obtain the asymptotics of the trace. Example 4.2 shows that even if $a_{j}(x)$ is constant, these coefficients cannot be integrated to yield the trace asymptotics.

Despite these obstacles, we prove that an asymptotic expansion for the trace of the basic heat operator exists. Let $\bar{q}$ be the minimum codimension of the leaf closures of $(M, \mathcal{F})$. As $t \rightarrow 0$, the trace $K_{B}(t)$ of the basic heat kernel on functions satisfies the following asymptotic expansion for any positive integer $J$ :

$$
K_{B}(t)=\frac{1}{(4 \pi t)^{\bar{q} / 2}}\left(a_{0}+\sum_{j>0,0 \leq k \leq K_{0}} a_{j k} t^{j / 2}(\log t)^{k}+\mathcal{O}\left(t^{\frac{J+1}{2}}(\log t)^{K_{0}-1}\right)\right),
$$

where $K_{0}$ is less than or equal to the number of different dimensions of leaf closures in $\mathcal{F}$, and where

$$
a_{0}=V_{t r}=\int_{M} \frac{1}{\operatorname{Vol}\left(\overline{L_{x}}\right)} \operatorname{dvol}(x)
$$

This is the content of Theorem 2.3 . If the codimension of $\mathcal{F}$ is less than 4 , then the logarithmic terms vanish. The idea of proof is as follows. We rewrite the integral $K_{B}(t)=\int_{M} K_{B}(t, x, x) \operatorname{dvol}(x)$ in terms of an integral over $W \times S O(q)$, where $W$ is the basic manifold, an $S O(q)$-manifold associated to $(M, \mathcal{F})$. Then, we apply the results of [10]. In Corollary 2.4, we obtain the Weyl asymptotic formula for the eigenvalues of the basic Laplacian. 
In Section 3, we derive the first two nontrivial coefficients in the asymptotic expansion (1.6) in some special cases, including but not limited to all possible types of Riemannian foliations of codimension two or less. In each of these cases, the asymptotic formula contains no logarithmic terms. We conjecture (Conjecture 2.6) that the asymptotic expansion for the general case has the same features. In Section 3.1 we derive the asymptotics for the case in which all of the leaf closures have the same dimension, for any codimension. In this case, the leaf closure space is an orbifold, and en route to the result we obtain the asymptotics of the orbifold heat trace, which may be of independent interest. We find the asymptotics for the transversally orientable, codimension one case in Section 3.2, for the nonorientable codimension one case in Section 3.3, and for codimension two Riemannian foliations in Section 3.5. We remark that the codimension two case yields five possible types of asymptotic expansions. In Section 3.4, we show how to simplify the general case by subdividing the basic manifold into pieces, and this result is used in the calculations of Section 3.5. In Section 4 we demonstrate the asymptotic formulas in two examples of codimension two foliations.

We remark that these asymptotic expansions yield new results concerning the spectrum of the basic Laplacian. By Corollary 2.4, the eigenvalues of the basic Laplacian determine the minimum leaf closure codimension and the transverse volume $V_{t r}$ of the foliation. The results of Section 3 give more specific information in special cases. For example, if the leaf closure codimension is one, then the spectrum of the basic Laplacian determines the $L^{2}$ norm of the mean curvature of the leaf closure foliation. Therefore, the spectrum determines whether or not the leaf closure foliation is minimal.

In most cases considered in the paper, we assume that the foliations are transversally oriented for simplicity. In Section 5 , we describe the method for obtaining the asymptotics of the basic heat kernel on Riemannian foliations that are not transversally orientable.

\section{HEAT KERNELS AND OPERATORS ON THE BASIC MANIFOLD}

In this section we introduce some notation, recall some results contained in 47, and then use these results to obtain a formula for the trace of the basic heat kernel. Let $M$ be an $n$-dimensional, closed, connected, oriented Riemannian manifold without boundary, and let $\mathcal{F}$ be a transversally-oriented, codimension $q$ foliation on $M$ for which the metric is bundle-like. As in the Introduction, we let $\Delta_{B}$ denote the basic Laplacian, and we let $K_{B}(t, x, y)$ be the basic heat kernel on functions defined in (1.4).

Let $\widehat{M}$ be the oriented transverse orthonormal frame bundle of $(M, \mathcal{F})$, and let $\pi$ be the natural projection $\pi: \widehat{M} \longrightarrow M$. The manifold $\widehat{M}$ is a principal $S O(q)$-bundle over $M$. Given $\hat{x} \in \widehat{M}$, let $\hat{x} g$ denote the well-defined right action of $g \in S O(q)$ applied to $\hat{x}$. Associated to $\mathcal{F}$ is the lifted foliation $\widehat{\mathcal{F}}$ on $\widehat{M}$. The lifted foliation is transversally parallelizable, and the closures of the leaves are fibers of a fiber bundle $\rho: \widehat{M} \longrightarrow W$. The manifold $W$ is smooth and is called the basic manifold (see [36, pp. 105-108, p. 147ff.]). Let $\overline{\mathcal{F}}$ denote the foliation of $\widehat{M}$ by leaf closures of $\widehat{\mathcal{F}}$.

Endow $\widehat{M}$ with the metric $g^{M}+g^{S O(q)}$, where $g^{M}$ is the pullback of the metric on $M$, and $g^{S O(q)}$ is the standard, normalized, biinvariant metric on the fibers. By 
this, we mean that we use the transverse Levi-Civita connection (see [36, p. 80ff.]) to do the following. We calculate the inner product of two horizontal vectors in $T_{\widehat{x}} \widehat{M}$ by using $g^{M}$, and we calculate the inner product of two vertical vectors using $g^{S O(q)}$. We require that vertical vectors are orthogonal to horizontal vectors. This metric is bundle-like for both $(\widehat{M}, \widehat{\mathcal{F}})$ and $(\widehat{M}, \overline{\mathcal{F}})$. The transverse metric on $(\widehat{M}, \overline{\mathcal{F}})$ induces a well-defined Riemannian metric on $W$. The group $G=S O(q)$ acts by isometries on $W$ according to $\rho(\hat{x}) g:=\rho(\hat{x} g)$ for $g \in S O(q)$.

The volume form on $\widehat{M}$ can be written as $\operatorname{dvol}_{\overline{\mathcal{F}}} \rho^{*} \operatorname{dvol}_{W}$, where $\operatorname{dvol}_{\overline{\mathcal{F}}}$ is the volume form of any leaf closure and $\mathrm{dvol}_{W}$ is the volume form on the basic manifold $W$. Let $\phi: W \rightarrow \mathbb{R}$ be defined by taking $\phi(y)$ to be the volume of $\rho^{-1}(y)$. The function $\phi$ is obviously positive and is also smooth, since $\rho$ is a smooth Riemannian submersion (see the proof of a similar fact in [42, Proposition 1.1]). Let (, ) denote the pointwise inner product of forms, and let $\langle$,$\rangle denote the L^{2}$-inner product of forms. Then for all $\alpha, \gamma \in \Omega^{*}(W)$,

$$
\begin{aligned}
\left\langle\rho^{*} \alpha, \rho^{*} \gamma\right\rangle_{\widehat{M}} & =\int_{\widehat{M}}\left(\rho^{*} \alpha, \rho^{*} \gamma\right)_{\widehat{M}} \operatorname{dvol}_{\overline{\mathcal{F}}} \rho^{*} \operatorname{dvol}_{W} \\
& =\int_{\widehat{M}} \rho^{*}(\alpha, \gamma)_{W} \operatorname{dvol}_{\overline{\mathcal{F}}} \rho^{*} \operatorname{dvol}_{W} \\
& =\int_{W} \phi \cdot(\alpha, \gamma)_{W} \operatorname{dvol}_{W}=\langle\phi \alpha, \gamma\rangle_{W}
\end{aligned}
$$

We have used the fact that for a bundle-like metric, the pointwise inner product has the same action on basic forms as the pullback of the pointwise inner product on the local quotient manifold. For our foliation $(\widehat{M}, \overline{\mathcal{F}}), W$ is the (global) quotient manifold.

Let $\widehat{\Delta}_{B}$ denote the basic Laplacian associated to the lifted foliation on $\widehat{M}$, and let $\Delta_{W}$ denote the ordinary Laplacian on $W$ corresponding to the induced metric on $W$. Note that $\phi$ is invariant under the right action of $S O(q)$ on $W$, so we define the smooth function $\psi: M \rightarrow \mathbb{R}$ by $\pi^{*} \psi=\rho^{*} \phi$. Let $\left.\sigma\right\lrcorner$ denote the adjoint of the wedge product $\sigma \wedge$ for any form $\sigma$.

We define the elliptic operator $\widetilde{\Delta_{W}}: C^{\infty}(W) \rightarrow \mathbb{R}$ by

$$
\begin{aligned}
\widetilde{\Delta_{W}} & \left.=\Delta_{W}-\frac{1}{\phi}(d \phi)\right\lrcorner \circ d \\
& =-g^{i j} \partial_{i} \partial_{j}-b^{j} \partial_{j} \text { locally on } W,
\end{aligned}
$$

where $g=\left(g_{i j}\right)$ is the metric on $W$ in local coordinates, $\left(g^{i j}\right)=g^{-1}$, and $b^{j}=$ $\partial_{i} g^{i j}+g^{i j} \partial_{i}(\log (\phi \sqrt{\operatorname{det} g}))$. Let $K_{B}$ and $\widehat{K_{B}}$ denote the basic heat kernels on $M$ and $\widehat{M}$, respectively, and let $\widetilde{K_{W}}$ denote the heat kernel corresponding to $\widetilde{\Delta_{W}}$ on $W$. Then we have the following results (see [47, Theorems 1.1 and 2.4]):

(1) The following equation holds on $C^{\infty}(W)$ :

$$
\widehat{\Delta}_{B} \rho^{*}=\rho^{*} \widetilde{\Delta_{W}} \text {. }
$$

(2) For every $x, y \in M, \hat{x} \in \pi^{-1}(x)$, and $\hat{y} \in \pi^{-1}(y)$,

$$
K_{B}(t, x, y)=\int_{G} \widetilde{K_{B}}(t, \hat{x}, \hat{y} g) \chi(g) .
$$


(3) For every $\hat{x} \in \pi^{-1}(x)$ and $\hat{y} \in \pi^{-1}(y)$,

$$
\widehat{K_{B}}(t, \hat{x}, \hat{y})=\frac{\widetilde{K_{W}}(t, \rho(\hat{x}), \rho(\hat{y}))}{\phi(\rho(\hat{y}))} .
$$

We will now write the trace of the basic heat kernel in terms of $\widetilde{K_{W}}$. Let dvol, $\widehat{\mathrm{dvol}}, \operatorname{dvol}_{W}$, and $\chi$ denote the volume forms on $M, \widehat{M}, W$, and $G=S O(q)$, respectively. Then

$$
\begin{aligned}
K_{B}(t) & =\operatorname{trace} e^{-t \Delta_{B}} \\
& =\int_{M} K_{B}(t, x, x) \mathrm{dvol} \\
& =\int_{M} \int_{G} \widehat{K_{B}}(t, \hat{x}, \hat{x} g) \chi(g) \operatorname{dvol}(x) .
\end{aligned}
$$

For any measurable section $s: M \rightarrow \widehat{M}$ that is smooth on an open, dense subset of $M$, we can describe points of $\widehat{M}$ in terms of the map $M \times G \rightarrow \widehat{M}$ defined by $(x, h) \mapsto s(x) h$. In these "coordinates," the measure on $\widehat{M}$ is $\widehat{\operatorname{dvol}}(x, h)=$ $\operatorname{dvol}(x) \chi(h)$. Therefore, if we change coordinates $\hat{x} \mapsto \hat{x} h$ in (2.4), average over $G$, and use Fubini's Theorem, we get

$$
\begin{aligned}
K_{B}(t) & =\int_{G} \int_{G} \int_{M} \widehat{K_{B}}(t, \hat{x} h, \hat{x} h g) \operatorname{dvol}(x) \chi(h) \chi(g) \\
& =\int_{G}\left\langle\widehat{K_{B}}(t, \cdot, \cdot g), 1\right\rangle_{\widehat{M}} \chi(g) \\
& =\int_{G}\left\langle\frac{\widehat{K_{W}}(t, \rho(\cdot), \rho(\cdot) g)}{\phi(\rho(\cdot))}, 1\right\rangle_{\widehat{M}} \chi(g) \text { by (2.3) } \\
& =\int_{G}\left\langle\widetilde{K_{W}}(t, \cdot, \cdot g), 1\right\rangle_{W} \chi(g) \quad \text { by (2.1) } \\
& =\int_{G \times W} \widetilde{K_{W}}(t, w, w g) \operatorname{dvol}_{W}(w) \chi(g) .
\end{aligned}
$$

We have shown the following:

Proposition 2.1. The trace $K_{B}(t)$ of the basic heat kernel on functions is given by the formula

$$
K_{B}(t)=\int_{G \times W} \widetilde{K_{W}}(t, w, w g) \operatorname{dvol}_{W}(w) \chi(g) .
$$

Corollary 2.2. The trace of the basic heat kernel on functions on $M$ is the same as the trace of the heat kernel corresponding to $\widetilde{\Delta_{W}}$ restricted to $S O(q)$-invariant functions on $W$.

Therefore, the results of [10] apply, since $\widetilde{\Delta_{W}}$ has the same principal symbol as $\Delta_{W}$ and commutes with the $G$-action. In particular, formula (1.3) holds, where $m=\operatorname{dim} W / G, K_{0}$ is less than or equal to the number of different dimensions of $G$-orbits in $W$, and $a_{0}=\operatorname{Vol}(W / G)$.

The leaf closures of $(M, \mathcal{F})$ with maximal dimension form an open, dense subset $M_{0}$ of $M$ (see [36, pp. 157-159]). The leaf closures of the lifted foliation cover the leaf closures of $M$ (see [36, p. 151ff.]). Given a leaf closure $\overline{L_{x}}$ containing $x \in M$, the dimension of a leaf closure contained in $\pi^{-1}\left(\overline{L_{x}}\right)$ is $\operatorname{dim} \overline{L_{x}}+\operatorname{dim} H_{\hat{x}}$, where 
$\hat{x} \in \pi^{-1}(x)$ and $H_{\hat{x}}$ is the subgroup of $S O(q)$ that fixes the leaf closure containing $\hat{x}$. In some sense, the group $H_{\hat{x}}$ measures the holonomy of the leaves contained in the leaf closure containing $x$ as well as the holonomy of the leaf closure. The group $H_{\hat{x}}$ is isomorphic to the structure group corresponding to the principal bundle $\bar{L}_{\hat{x}} \rightarrow \bar{L}_{x}$, where $\bar{L}_{\hat{x}}$ is the leaf closure in $\widehat{M}$ that contains $\hat{x}$; the conjugacy class of $H \subset G$ depends only on the leaf $L_{x}$. Therefore, on an open, dense subset of $W$, the orbits of $G$ have dimension $\frac{q(q-1)}{2}-m$, where $m$ is the dimension of the principal isotropy groups. The above discussion also implies that the dimension of $W$ is $\bar{q}+\frac{q(q-1)}{2}-m$, where $\bar{q}$ is the codimension of the leaf closures of $M$ of maximal dimension. As a result, we have that

$$
m=\operatorname{dim} W / G=\operatorname{dim} W-\left(\frac{q(q-1)}{2}-m\right)=\bar{q} .
$$

Also, the number of different dimensions of $G$-orbits in $W$ is equal to the number of different dimensions of leaf closures in $(M, \mathcal{F})$.

For $w \in W$, let $w G$ denote the $G$-orbit of $w$. By construction,

$$
\begin{aligned}
\operatorname{Vol}(w G) \cdot \phi(w) & =\operatorname{Vol}\left(\rho^{-1}(w G)\right) \\
& =\operatorname{Vol}\left(\overline{L_{x}}\right),
\end{aligned}
$$

where $x$ is chosen so that $x \in \pi\left(\rho^{-1}(w G)\right)$. Using the above information, we have

$$
\begin{aligned}
\operatorname{Vol}(W / G) & =\int_{W} \frac{1}{\operatorname{Vol}(w G)} \operatorname{dvol}_{W}(w) \\
& =\int_{W} \frac{\phi(w)}{\operatorname{Vol}\left(\rho^{-1}(w G)\right)} \operatorname{dvol}_{W}(w) \\
& =\int_{\widehat{M}} \frac{1}{\operatorname{Vol}\left(\rho^{-1}(\rho(\hat{x}) G)\right)} \widehat{\operatorname{dvol}(\hat{x}) \quad \text { by (2.1) }} \\
& =\int_{\widehat{M}} \frac{1}{\operatorname{Vol}\left(\overline{L_{\pi(\hat{x})}}\right)} \widehat{\operatorname{dvol}(\hat{x})} \\
& =\int_{M} \frac{1}{\operatorname{Vol}\left(\overline{L_{x}}\right)} \operatorname{dvol}(x) .
\end{aligned}
$$

We remark that the integrals above converge. Proposition 2.1 in [4], which concerns isometric flows, is easily modified to show that the first integral above converges; the convergence of the other integrals follows.

Using the above discussions and the results of [10, we have the following:

Theorem 2.3. Let $\bar{q}$ be the codimension of the leaf closures of $(M, \mathcal{F})$ with maximal dimension. As $t \rightarrow 0$, the trace $K_{B}(t)$ of the basic heat kernel on functions satisfies the following asymptotic expansion for any positive integer $J$ :

$$
K_{B}(t)=\frac{1}{(4 \pi t)^{\bar{q} / 2}}\left(a_{0}+\sum_{j>0,0 \leq k \leq K_{0}} a_{j k} t^{j / 2}(\log t)^{k}+\mathcal{O}\left(t^{\frac{J+1}{2}}(\log t)^{K_{0}-1}\right)\right),
$$

where $K_{0}$ is less than or equal to the number of different dimensions of leaf closures of $(M, \mathcal{F})$, and where

$$
a_{0}=V_{t r}=\int_{M} \frac{1}{\operatorname{Vol}\left(\overline{L_{x}}\right)} \operatorname{dvol}(x)
$$


The coefficients of the logarithmic terms in the expansion vanish if the codimension of the foliation is less than four.

In the last statement of the above proposition, we used the fact that $S O(q)$ is connected, has rank one for $q<4$, and acts effectively on $W$. Also, note that $a_{j k}$ depends only on the transverse metric, because by the results in [10] it depends only on infinitesimal metric information on the set $\{(g, w) \mid w g=w\}$, which is entirely determined by the transverse geometry of $(M, \mathcal{F})$.

We will call the coefficients $a_{0}$ and $a_{j k}$ the basic heat invariants; they are functions of the spectrum of the basic Laplacian because of the formula (see [42, Theorem 3.5])

$$
K_{B}(t)=\operatorname{trace}\left(e^{-t \Delta_{B}}\right)=\sum_{j \geq 0} e^{-t \lambda_{j}^{B}} .
$$

Using Karamata's theorem ([24, pp. 418-423]), we also have the following, which generalizes Weyl's asymptotic formula ([51]):

Corollary 2.4. Let $0=\lambda_{0}<\lambda_{1}^{B} \leq \lambda_{2}^{B} \leq \ldots$ be the eigenvalues of the basic Laplacian on functions, counting multiplicities. Then the spectral counting function $N_{B}(\lambda)$ satisfies the following asymptotic formula:

$$
\begin{aligned}
N_{B}(\lambda) & :=\#\left\{\lambda_{m}^{B} \mid \lambda_{m}^{B} \leq \lambda\right\} \\
& \sim \frac{V_{t r}}{(4 \pi)^{\bar{q} / 2} \Gamma\left(\frac{\bar{q}}{2}+1\right)} \lambda^{\bar{q} / 2}
\end{aligned}
$$

as $\lambda \rightarrow \infty$, where $\bar{q}$ and $V_{\text {tr }}$ are defined as above.

Observe that we are able to prove Theorem 2.3 and Corollary 2.4 with very little information about the heat kernel $\widetilde{K_{W}}$; we used Proposition 2.1 and the results in [10] alone. We also remark that although the expansion (1.3) contains logarithmic terms, no examples for which these terms are nonzero are known. In the proof of this expansion [10, the authors show that $G \times M$ can be decomposed into pieces over which the integral has an expansion with possibly nonzero logarithmic terms. In the cases for which the authors proved the nonexistence of logarithmic terms, symmetries cause the sum of these logarithmic contributions to vanish. We make the following conjectures:

Conjecture 2.5. Suppose that $\Gamma$ is a compact group that acts isometrically and effectively on a compact, connected Riemannian manifold $M$. Then the coefficients $a_{j k}$ of the equivariant trace formula (1.3) satisfy the following:

- $a_{j k}=0$ for $k>0$.

- If $M$ is oriented and $\Gamma$ acts by orientation-preserving isometries, then $a_{j 0}=$ 0 for $j$ odd.

Conjecture 2.6 (Corollary of Conjecture 2.5). In Theorem 2.3, $a_{j k}=0$ for $k>0$. If in addition $S O(q)$ acts by orientation-preserving isometries on $W$, then $a_{j 0}=0$ for $j$ odd.

We remark that since our foliation is transversally oriented, $S O(q)$ acts by orientation-preserving isometries precisely when the leaf closures are all transversally orientable. The $S O(q)$ action is not always orientation-preserving, as Example 4.2 shows. 


\section{Formulas FOR THE BASIC HEAT INVARIANTS IN SPECIAL CASES}

We will now explicitly derive the asymptotics of the integral in Proposition 2.1 in some special cases.

3.1. Regular closure. Suppose that $(M, \mathcal{F})$ has regular closure. In other words, assume that the leaf closures all have the same dimension. Note that this implies that all of the leaves and leaf closures have finite holonomy. In this case, the orbits of $S O(q)$ or $O(q)$ (and thus the leaf closures of $(M, \mathcal{F})$ ) all have the same dimension, and the space of leaf closures is a Riemannian orbifold. Since the orbits all have the same dimension, locally defined functions of the metric along the orbits are smooth (bounded) functions on the basic manifold, and the volumes of the orbits (and hence the volumes of the leaf closures) are bounded away from zero. Therefore, the coefficients in the asymptotic expansion for $K_{B}(t, x, x)$ found in 47 are bounded on the foliated manifold $M$, but the error term is not necessarily bounded. These local expressions cannot in general be integrated over $M$ to yield the asymptotics of the trace of the basic heat operator, as in the method used to obtain the asymptotic expansion of the trace of the ordinary heat operator on a manifold, as described in the Introduction. Instead, a calculation of the trace of a second order operator on an orbifold is required.

Note that the leaf closures of $(M, \mathcal{F})$ themselves form a Riemannian foliation $\left(M, \mathcal{F}^{c}\right)$, in which all leaves are compact. In general the basic Laplacian $\Delta_{B}$ on functions satisfies $\Delta_{B}=P_{B} \delta d$, where $d$ is the exterior derivative, $\delta$ is the $L^{2}$ adjoint of $d$, and $P_{B}$ is the orthogonal projection of $L^{2}$ functions to the $L^{2}$ of basic functions (see 42]). Since the projection $P_{B}$ on functions is identical for both foliations $(M, \mathcal{F})$ and $\left(M, \mathcal{F}^{c}\right)$, the basic Laplacian on basic functions of the foliation $(M, \mathcal{F})$ is the same as the basic Laplacian $\Delta_{B}^{c}$ on basic functions of the foliation $\left(M, \mathcal{F}^{c}\right)$. Note that the equivalent statement for the basic Laplacian on forms is false.

Thus, it suffices to solve the problem of calculating the basic heat kernel asymptotics for the case of closed leaves. Let $p: M \rightarrow N=M / \mathcal{F}^{c}$ be the quotient map, which is a Riemannian submersion away from the leaf closures with holonomy. Similar to the arguments in Section 2, the basic Laplacian on functions satisfies

$$
\left.\Delta_{B} \circ p^{*}=p^{*} \circ\left(\Delta^{N}-\frac{d \psi}{\psi}\right\lrcorner \circ d\right),
$$

where $\Delta^{N}=\delta d$ is the ordinary Laplacian on the orbifold $N$, and $\psi$ is the function on $N$ defined by $\psi(x)=\operatorname{Vol}\left(p^{-1}(x)\right)$ if $p^{-1}(x)$ is a principal leaf closure and extended to be continuous (and smooth) on $N$. Note that if $\kappa^{c}$ is the mean curvature form of $\left(M, \mathcal{F}^{c}\right)$ and $P_{B}^{1}$ is the $L^{2}$ projection from one-forms to basic one-forms, then $P_{B}^{1} \kappa^{c}=-p^{*}\left(\frac{d \psi}{\psi}\right)$. Since $f$ is a basic function on $M$ if and only if $f=p^{*} g$ for some function $g$ on $N$, the trace of $K_{B}(t)$ of the basic heat kernel on functions on $M$ is the trace of $e^{\left.-t\left(\Delta^{N}-\frac{d \psi}{\psi}\right\lrcorner \circ d\right)}$ on functions on the orbifold $N$. To calculate this trace, we first collect the following known results.

Lemma 3.1 (See [14, 27], 49]). Let L be a second order operator on functions on a closed Riemannian manifold $N$ of dimension $m$, such that $L=\Delta+V+Z$, where $\Delta$ is the Laplace operator, $V$ is a purely first order operator, and $Z$ is a zeroth order 
operator. Then, the heat kernel $K_{L}(t, x, y)$ of $L$, the fundamental solution of the operator $\frac{\partial}{\partial t}+L$, exists and has the following properties:

(1) Given $\varepsilon>0$, there exists $c>0$ such that if $r(x, y)=\operatorname{dist}(x, y)>\varepsilon$, then $K_{L}(t, x, y)=\mathcal{O}\left(e^{-c / t}\right)$ as $t \rightarrow 0$.

(2) If $r(x, y)=\operatorname{dist}(x, y)$ is sufficiently small, then as $t \rightarrow 0$,

$$
K_{L}(t, x, y)=\frac{e^{-r^{2}(x, y) / 4 t}}{(4 \pi t)^{m / 2}}\left(c_{0}(x, y)+c_{1}(x, y) t+\ldots+c_{k}(x, y)+\mathcal{O}\left(t^{k+1}\right)\right)
$$

for any $k$, each $c_{j}(x, y)$ is smooth, and $c_{0}(x, x)=1$. The function $c_{j}(x, y)$ is determined by the metric and the operators $V$ and $Z$ and their derivatives, evaluated along the minimal geodesic connecting $x$ and $y$.

If the manifold $N$ is instead a Riemannian orbifold, the operators can still be defined (by their definitions on the local covers using pullbacks), and a fundamental solution to the heat equation still exists. Note that in all such cases, the lifted operator $\widetilde{L}=\widetilde{\Delta}+\widetilde{V}+\widetilde{Z}$ is equivariant with respect to the local finite group action. Since the asymptotics of the heat kernel are still determined locally, we have the following lemma.

Lemma 3.2. Let $L$ be a second order operator on functions on a closed Riemannian orbifold $N$ of dimension $m$, such that $L=\Delta+V+Z$, where $\Delta$ is the Laplace operator, $V$ is a purely first order operator, and $Z$ is a zeroth order operator. Then, the heat kernel $K_{L}(t, x, y)$ of $L$, the fundamental solution of the operator $\frac{\partial}{\partial t}+L$, exists and has the following properties:

(1) Given $\varepsilon>0$, there exists $c>0$ such that if $r(x, y)=\operatorname{dist}(x, y)>\varepsilon$, then $K_{L}(t, x, y)=\mathcal{O}\left(e^{-c / t}\right)$ as $t \rightarrow 0$.

(2) If $r(x, y)=\operatorname{dist}(x, y)$ is sufficiently small, then as $t \rightarrow 0$, and if the minimal geodesic connecting $x$ and $y$ is away from the singular set of the orbifold, then

$$
K_{L}(t, x, y)=\frac{e^{-r^{2}(x, y) / 4 t}}{(4 \pi t)^{m / 2}}\left(c_{0}(x, y)+c_{1}(x, y) t+\ldots+c_{k}(x, y)+\mathcal{O}\left(t^{k+1}\right)\right)
$$

for any $k$, each $c_{j}(x, y)$ is smooth, and $c_{0}(x, x)=1$.

(3) Let $z$ be an element of the singular set of $N$, and let $H_{z}$ denote the finite group of isometries such that a neighborhood $U$ of $z$ in $N$ is isometric to $\widetilde{U} / H_{z}$, where $\widetilde{U}$ is an open set in $\mathbb{R}^{m}$ with the given metric. Let o $\left(H_{z}\right)$ denote the order of $H_{z}$. Assuming that the neighborhood $U$ is sufficiently small, there exists $c>0$ such that if $x, y \in U$, then

$$
K_{L}(t, x, y)=\frac{1}{o\left(H_{z}\right)} \sum_{h \in H_{z}} \widetilde{K_{L}}(t, x, h y)+\mathcal{O}\left(e^{-c / t}\right)
$$

where $\widetilde{K_{L}}$ is the heat kernel of the lifted operator $\widetilde{\Delta}+\widetilde{V}+\widetilde{Z}$ on $\widetilde{U}$, which itself satisfies an asymptotic expansion as in (3.1) above. 
The results above are well-known and well-utilized in the cases where $V=Z=0$ (see, for example, [17, [18]), but they are true in the generality stated.

Next, we establish an estimate and a trigonometric identity.

Lemma 3.3. Given $a>0$ and $b \in \mathbb{N}$, we have

$$
\begin{aligned}
\int_{0}^{\varepsilon} e^{-\frac{x^{2} a^{2}}{t}} x^{b} d x & =\int_{0}^{\infty} e^{-\frac{x^{2} a^{2}}{t}} x^{b} d x-\int_{\varepsilon}^{\infty} e^{-\frac{x^{2} a^{2}}{t}} x^{b} d x \\
& =\frac{\Gamma\left(\frac{b+1}{2}\right)}{2 a^{b+1}} t^{\frac{b+1}{2}}+\mathcal{O}\left(\left(\frac{\varepsilon^{2} a^{2}}{t}\right)^{\frac{b-1}{2}} e^{-\frac{\varepsilon^{2} a^{2}}{t}}\right) \\
& =\frac{\Gamma\left(\frac{b+1}{2}\right)}{2 a^{b+1}} t^{\frac{b+1}{2}}+\mathcal{O}\left(\left(\frac{t}{\varepsilon^{2} a^{2}}\right)^{N}\right) \text { as } t \rightarrow 0,
\end{aligned}
$$

for any $N \geq \frac{b+1}{2}$.

Proof. Substituting $u=\frac{x^{2} a^{2}}{t}$ or $x=\frac{\sqrt{u t}}{a}$, we get

$$
\begin{aligned}
\int_{0}^{\varepsilon} e^{-\frac{x^{2} a^{2}}{t}} x^{b} d x & =\frac{t^{\frac{b+1}{2}}}{2 a^{b+1}} \int_{0}^{\varepsilon^{2} a^{2} / t} e^{-u} u^{\frac{b-1}{2}} d u \\
& =\frac{t^{\frac{b+1}{2}}}{2 a^{b+1}} \int_{0}^{\infty} e^{-u} u^{\frac{b-1}{2}} d u-\frac{t^{\frac{b+1}{2}}}{2 a^{b+1}} \int_{\varepsilon^{2} a^{2} / t}^{\infty} e^{-u} u^{\frac{b-1}{2}} d u \\
& =\frac{t^{\frac{b+1}{2}} \Gamma\left(\frac{b+1}{2}\right)}{2 a^{b+1}}-\frac{t^{\frac{b+1}{2}}}{2 a^{b+1}} \Gamma\left(\frac{b+1}{2}, \frac{\varepsilon^{2} a^{2}}{t}\right),
\end{aligned}
$$

where $\Gamma(A, z)$ is the (upper) incomplete Gamma function. It is known that $\Gamma(A, z)$ is proportional to $e^{-z} z^{A-1}\left(1+\mathcal{O}\left(\frac{1}{z}\right)\right)$ as $|z| \rightarrow \infty$, and the formulas above follow.

Lemma 3.4. For any positive integer $k$,

$$
\sum_{j=1}^{k-1} \frac{1}{\sin ^{2}\left(\frac{\pi j}{k}\right)}=\frac{k^{2}-1}{3} .
$$

Proof. Many thanks to George Gilbert. Contact the author for a proof.

The goal is to evaluate the asymptotics of

$$
K_{B}(t)=\operatorname{tr}\left(\left.e^{-t P}\right|_{C^{\infty}(N)}\right)=\int_{N} K_{P}(t, x, x) \text { dvol }
$$

as $t \rightarrow 0$, where $\left.P=\Delta^{N}-\frac{d \psi}{\psi}\right\lrcorner \circ d$, but we first proceed with calculating the heat trace of a general operator $L$ as in (3.1) on an orbifold. We now decompose $N$ as follows. Given an element $z \in N$, let $H_{z}$ denote a subgroup of the orthogonal group $O(\operatorname{dim} N)$ such that every sufficiently small metric ball around $z$ is isometric to a ball in $H_{z} \backslash\left(\mathbb{R}^{\operatorname{dim} N}, g\right)$, where $g$ is an $H_{z}$-invariant metric. The conjugacy class $\left[H_{z}\right]$ in $O(\operatorname{dim} N)$ is called an isotropy type of $z$. The stratification of $N$ is a partition of $N$ into the different isotropy types. The partial order on these isotropy types is defined as in the general $G$-manifold structure (see Section 3.4). Let $o\left(H_{z}\right)$ denote the order of $H_{z}$. 
As in Section 3.4, we decompose $N$ into pieces which include tubular neighborhoods of parts of the singular strata of the orbifold and the principal stratum (for which $H_{z}=\{e\}$ ) minus the other neighborhoods. We may further decompose $N=N_{\varepsilon} \cup \amalg N_{j}$ as a finite disjoint union, where each $N_{j}$ is of the form $H_{j} \backslash \widetilde{N}_{j}$ with $\widetilde{N_{j}}$ contractible, no nontrivial element of $H_{j}$ fixing all of $\widetilde{N_{j}}$, and with at least one point of $\widetilde{N_{j}}$ having isotropy $H_{j}$. We may think of $N_{j}$ as a tubular neighborhood of an open subset of a singular stratum, up to sets of measure zero.

Given any isometry $h \in H_{i} \backslash\{e\}$, choose a tubular neighborhood

$$
U_{h, \varepsilon}^{i}=T_{\varepsilon}\left(H_{j} \backslash\left(\widetilde{N_{i}}\right)^{h}\right) \cap N_{i}
$$

of the local submanifold $S_{i}^{h}=\left(H_{j} \backslash \widetilde{N}_{i}\right)^{h}$ of singular points fixed by $h$, and let $\widetilde{U_{h, \varepsilon}^{i}}=T_{\varepsilon}\left(\left(\widetilde{N_{i}}\right)^{h}\right) \cap \widetilde{N}_{i}$ denote the local cover. Let $\widetilde{U_{e, \varepsilon}^{i}}=\widetilde{N_{i}}$.

$$
N_{\varepsilon}=N \backslash \bigcup_{i}\left(\bigcup_{h \in H_{i} \backslash\{e\}} U_{h, \varepsilon}^{i}\right) .
$$

Then there exists $c>0$ such that

$$
\begin{gathered}
K_{L}(t)+\mathcal{O}\left(e^{-c / t}\right) \\
=\int_{N_{\varepsilon}} K_{L}(t, x, x) \mathrm{dvol}+\sum_{i} \frac{1}{o\left(H_{i}\right)} \sum_{h \in H_{i}} \int_{\widetilde{U_{h, \varepsilon}^{i}}} \widetilde{K_{L}}(t, x, h x) \mathrm{dvol} \\
=\frac{1}{(4 \pi t)^{m / 2}}\left(\int_{N_{\varepsilon}} \mathrm{dvol}+t \int_{N_{\varepsilon}} c_{1}(x, x) \mathrm{dvol}+\mathcal{O}\left(t^{2}\right)\right) \\
+\sum_{i} \frac{1}{o\left(H_{i}\right)}\left(\int_{\widetilde{N_{i}}} \mathrm{dvol}+t \int_{\widetilde{N_{i}}} c_{1}(x, x) \mathrm{dvol}+\mathcal{O}\left(t^{2}\right)\right) \\
+\sum_{i} \frac{1}{o\left(H_{i}\right)(4 \pi t)^{m / 2}} \sum_{h \in H_{i} \backslash\{e\}} \int_{\widetilde{U_{h, \varepsilon}^{i}}} e^{-r^{2}(x, h x) / 4 t}\left(c_{0}(x, h x)+\mathcal{O}\left(t^{1}\right)\right) \mathrm{dvol} .
\end{gathered}
$$

Note that if $h \neq e, S_{i}^{h}$ is a disjoint union of connected submanifolds $S_{i, j}^{h}$ codimension $d_{h}^{i, j}>0$. Then, following a calculation in [17, we may rewrite the last integral in geodesic normal coordinates $x$. If $B_{\varepsilon}^{i, j}(y)$ denotes the normal exponential ball of radius $\varepsilon$ at $y \in \widetilde{S_{i, j}^{h}}$, its volume form $\operatorname{dvol}_{B_{\varepsilon}^{i, j}}$ satisfies $\operatorname{dvol}_{B_{\varepsilon}^{i, j}}=\left(1+\mathcal{O}\left(|x|^{2}\right)\right) d x$, and the volume form dvol on $\widetilde{U_{h, \varepsilon}^{i}}$ satisfies dvol $=\left(1+\mathcal{O}\left(|x|^{2}\right)\right) \operatorname{dvol}_{B_{\varepsilon}^{i, j}} \operatorname{dvol}_{\widetilde{S_{i, j}^{h}}}$ 
for each $j$.

$$
\begin{aligned}
& \int_{\widetilde{U_{h, \varepsilon}^{i}}} e^{-r^{2}(x, h x) / 4 t}\left(c_{0}(x, h x)+\mathcal{O}\left(t^{1}\right)\right) \mathrm{dvol} \\
&= \sum_{j} \int_{\widetilde{S_{i, j}^{h}}} \int_{B_{\varepsilon}^{i, j}} e^{-r^{2}(x, h x) / 4 t}\left(1+\mathcal{O}\left(r^{2}\right)+\mathcal{O}(t)\right) \mathrm{dvol}_{B_{\varepsilon}^{i, j}} \mathrm{dvol}_{\overparen{S_{i, j}^{h}}} \\
&= \sum_{j} \int_{\widetilde{S_{i, j}^{h}}} \int_{B_{\varepsilon}^{i, j}} e^{-r^{2}(x, h x) / 4 t}\left(1+\mathcal{O}\left(|x|^{2}\right)+\mathcal{O}(t)\right) d x \operatorname{dvol}_{\widehat{S_{i, j}^{h}}} \\
&= \sum_{j} \int_{\widetilde{S_{i, j}^{h}}} \int_{(I-h) B_{\varepsilon}^{i, j}} e^{-r^{2}(u+h x(u), h x(u)) / 4 t}\left(1+\mathcal{O}\left(|u|^{2}\right)+\mathcal{O}(t)\right) \\
& \cdot\left|\operatorname{det}(I-h)^{-1}\right| d u \operatorname{dvol}_{\overparen{S_{i, j}^{h}}},
\end{aligned}
$$

using the change of variables $u=(I-h) x$. Further (see [17]), there is a change of variables $y(u)$ such that $u_{j}=y_{j}+\mathcal{O}\left(|y|^{3}\right)$ and $r^{2}(u+h x(u), h x(u))=\sum y_{j}^{2}$, and the Jacobian for this change of variables is $1+\mathcal{O}\left(|y|^{2}\right)$. Thus,

$$
\begin{aligned}
& \int_{\widetilde{U_{h, \varepsilon}^{i}}} e^{-r^{2}(x, h x) / 4 t}\left(c_{0}(x, h x)+\mathcal{O}\left(t^{1}\right)\right) \text { dvol } \\
&=\left|\operatorname{det}(I-h)^{-1}\right| \sum_{j} \int_{\widetilde{S_{i, j}^{h}}} \int_{y\left((I-h) B_{\varepsilon}^{i, j}\right)} e^{-|y|^{2} / 4 t} \\
& \cdot\left(1+\mathcal{O}\left(|y|^{2}\right)+\mathcal{O}(t)\right) d y \operatorname{dvol}_{\widetilde{S_{i, j}^{h}}} .
\end{aligned}
$$

By Lemma 3.3, we have

$$
\begin{aligned}
\int_{y\left((I-h) B_{\varepsilon}^{i, j}\right)} & e^{-|y|^{2} / 4 t}\left(1+\mathcal{O}\left(|y|^{2}\right)+\mathcal{O}(t)\right) d y \\
= & \int_{\mathbb{R}^{d_{h}^{i, j}}} e^{-|y|^{2} / 4 t} d y+\mathcal{O}\left(t^{\left(d_{z}+2\right) / 2}\right) \\
= & \left(2 \cdot \frac{\Gamma\left(\frac{1}{2}\right)}{2\left(\frac{1}{2}\right)}\right)^{d_{h}^{i, j}} t^{d_{h}^{i, j} / 2}+\mathcal{O}\left(t^{\left(d_{h}^{i, j}+2\right) / 2}\right) \\
= & (4 \pi t)^{d_{h}^{i, j} / 2}+\mathcal{O}\left(t^{\left(d_{h}^{i, j}+2\right) / 2}\right) .
\end{aligned}
$$

Thus,

$$
\begin{aligned}
\int_{\widetilde{U_{h, \varepsilon}^{i}}} e^{-r^{2}(x, h x) / 4 t} & \left(c_{0}(x, h x)+\mathcal{O}\left(t^{1}\right)\right) \text { dvol } \\
= & \left|\operatorname{det}(I-h)^{-1}\right| \sum_{j} \operatorname{vol}\left(\widetilde{S_{i, j}^{h}}\right)(4 \pi t)^{d_{h}^{i, j} / 2}+\mathcal{O}\left(t^{\left(d_{h}^{i, j}+2\right) / 2}\right) .
\end{aligned}
$$


Hence, letting $\varepsilon$ approach zero and summing up over the neighborhoods of the singular strata of the orbifold $N$, we have

$$
\begin{gathered}
K_{L}(t)=\frac{1}{(4 \pi t)^{m / 2}}\left(\int_{N} \operatorname{dvol}+t \int_{N} c_{1}(x, x) \operatorname{dvol}\right. \\
\left.+\mathcal{O}\left(t^{2}\right)\right) \\
+\frac{1}{(4 \pi t)^{m / 2}} \sum_{i} \frac{1}{o\left(H_{i}\right)} \sum_{h \in H_{i} \backslash\{e\}} \sum_{j}\left|\operatorname{det}(I-h)^{-1}\right| \operatorname{vol}\left(\widetilde{S_{i, j}^{h}}\right)(4 \pi t)^{d_{h}^{i, j} / 2} \\
+\mathcal{O}\left(t^{\left(d_{h}^{i, j}+2\right) / 2}\right) \\
=\frac{1}{(4 \pi t)^{m / 2}}\left(\operatorname{vol}(N)+t \int_{N} c_{1}(x, x) \operatorname{dvol}\right) \\
+\frac{1}{(4 \pi t)^{m / 2}}\left(\sum_{i} \frac{1}{o\left(H_{i}\right)} \sum_{h \in H_{i} \backslash\{e\}} \sum_{d_{h}^{i, j}=1,2}\left|\operatorname{det}(I-h)^{-1}\right| \operatorname{vol}\left(\widetilde{S_{i, j}^{h}}\right)(4 \pi t)^{d_{h}^{i, j} / 2}\right) \\
+\mathcal{O}\left(t^{(3-m) / 2}\right) .
\end{gathered}
$$

Note that $\widetilde{S_{i, j}^{h}}$ has codimension 1 precisely when $h$ acts as a reflection, in which case $\left|\operatorname{det}(I-h)^{-1}\right|=(1-(-1))^{-1}=\frac{1}{2}$. Similarly, $\widetilde{S_{i, j}^{h}}$ has codimension 2 exactly when $h$ acts as a rotation (say by $\theta_{h}=\frac{2 \pi}{k}$ for some $k \in \mathbb{Z}_{>0}$ ) in the normal space to $\widetilde{S_{i, j}^{h}}$. In that case,

$$
\begin{aligned}
\left|\operatorname{det}(I-h)^{-1}\right|=\left|\operatorname{det}\left(\begin{array}{cc}
1-\cos \theta_{h} & -\sin \theta_{h} \\
\sin \theta_{h} & 1-\cos \theta_{h}
\end{array}\right)^{-1}\right| \\
=\frac{1}{2-2 \cos \left(\theta_{h}\right)}=\frac{1}{4 \sin ^{2}\left(\frac{\theta_{h}}{2}\right)} .
\end{aligned}
$$

If this number is summed over all nontrivial elements of a cyclic group group of rotations by $\left\{\frac{2 \pi}{k}, \frac{4 \pi}{k}, \ldots, \frac{2(k-1) \pi}{k}\right\}$, by Lemma 3.4 we have

$$
\sum_{j=1}^{k-1} \frac{1}{4 \sin ^{2}\left(\frac{\pi j}{k}\right)}=\frac{k^{2}-1}{12} .
$$

In each of these cases, generic points $z$ of $S_{i, j}^{h}$ have isotropy subgroups $H_{z}$ isomorphic to a cyclic group.

To obtain the asymptotic expansion, we identify two subsets of the singular part of the orbifold:

$$
\begin{aligned}
\Sigma_{\mathrm{ref}} N= & \left\{z \in N: H_{z} \text { has order } 2 \text { and is generated by a reflection }\right\} \\
\Sigma_{k} N= & \left\{z \in N: H_{z} \text { is a cyclic group of order } k\right. \\
& \text { and consists of rotations in a plane }\}
\end{aligned}
$$


Note that

$$
\begin{aligned}
\operatorname{vol}^{m-1}\left(N_{i} \cap \Sigma_{\text {ref }} N\right) & =\frac{2}{o\left(H_{i}\right)} \sum_{h \text { reflection } \operatorname{vol}^{m-1}\left(\widetilde{S_{i}^{h}}\right)}=\sum_{h \text { reflection }} \operatorname{vol}^{m-1}\left(S_{i}^{h}\right), \\
\operatorname{vol}^{m-2}\left(N_{i} \cap \Sigma_{k} N\right) & =\frac{k}{o\left(H_{i}\right)} \sum_{h \text { rotation of } \frac{2 \pi}{k}} \operatorname{vol}^{m-2}\left(\widetilde{S_{i}^{h}}\right) \\
\sum_{h \text { rotation of } \frac{2 \pi}{k}} \operatorname{vol}^{m-2}\left(S_{i}^{h}\right) & =\sum_{h \text { rotation by } \frac{2 \pi j}{k}} \operatorname{vol}^{m-2}\left(S_{i}^{h}\right) \text { for fixed } j .
\end{aligned}
$$

We now combine the results above with (3.2) to obtain the following theorem.

Theorem 3.5. Let $L$ be a second order operator on functions on a closed Riemannian orbifold $N$ of dimension $m$, such that $L=\Delta+V+Z$, where $\Delta$ is the Laplace operator, $V$ is a purely first order operator, and $Z$ is a zeroth order operator. Then, the trace of the heat operator has the following asymptotic expansion as $t \rightarrow 0$ :

$$
\begin{aligned}
K_{L}(t)= & \frac{1}{(4 \pi t)^{m / 2}}\left(\operatorname{vol}^{m}(N)+t^{1 / 2} \frac{\sqrt{\pi}}{2} \operatorname{vol}^{m-1}\left(\Sigma_{\mathrm{ref}} N\right)\right. \\
& \left.+t\left(\int_{N} c_{1}(x, x) \mathrm{dvol}+\frac{\pi\left(k^{2}-1\right)}{3 k} \mathrm{vol}^{m-2}\left(\Sigma_{k} N\right)\right)+\mathcal{O}\left(t^{3 / 2}\right)\right),
\end{aligned}
$$

where $c_{1}(x, x)$ is the heat trace coefficient from formula (3.1) and $\Sigma_{\mathrm{ref}} N$ and $\Sigma_{k} N$ are parts of the singular stratum of the orbifold defined in the paragraph above.

Note that the truth of this theorem is easily checked in the case of a manifold with boundary or with a manifold quotient by a finite cyclic group of rotations. Also, note that the coefficient $c_{1}(x, x)$ may be computed using standard methods as in [49.

We now wish to apply this result to the foliation case. Here, $N=M / \mathcal{F}^{c}$ is the leaf closure space (a Riemannian orbifold) of a Riemannian foliation $(M, \mathcal{F})$ with regular closure. The operator of note is

$$
K_{B}(t)=\operatorname{tr}\left(\left.e^{-t L}\right|_{C^{\infty}(N)}\right)=\int_{N} K_{L}(t, x, x) \text { dvol }
$$

as $t \rightarrow 0$, where $\left.L=\Delta^{N}-\frac{d \psi}{\psi}\right\lrcorner \circ d=\Delta^{N}+H^{c}$, and $H^{c}$ is the projection of the mean curvature vector field of the foliation of $M$ by leaf closures to the set of projectable vector fields, which implies that it descends to a vector field on $N$. The formula needed is $c_{1}(x, x)$; we refer to [47, formula (3.6)] for a similar calculation, which yields in our case

$$
\begin{aligned}
c_{1}(x, x) & =\frac{S(x)}{6}+\frac{\Delta^{N} \psi(x)}{2 \psi(x)}+\frac{1}{4}\left|H^{c}(x)\right|^{2} \\
& =\frac{S(x)}{6}+\frac{\Delta^{N} \psi(x)}{2 \psi(x)}+\frac{1}{4}\left|\frac{d \psi}{\psi}\right|^{2}(x),
\end{aligned}
$$

where $S(x)$ is the scalar curvature at $x \in N$. The theorem below follows. 
Theorem 3.6. Let $(M, \mathcal{F})$ be a Riemannian foliation with regular closure, so that the quotient orbifold $N=M / \mathcal{F}^{c}$ by leaf closures has dimension $m$. If $x \in N$ corresponds to a principal leaf closure, let $\psi(x)$ be the volume of the leaf closure, and extend this function to be smooth on $N$. Let $S$ denote the scalar curvature of $N$. Further, let $\Sigma_{\text {ref }} N$ be the set of singular points of $N$ corresponding to true boundary points, and let $\Sigma_{k} N$ be the set of singular points of $N$ which have neighborhoods diffeomorphic to $\mathbb{R}^{n}$ mod a planar cyclic group of rotations of order $k$. Then the trace of the basic heat kernel on functions satisfies the following asymptotic formula as $t \rightarrow 0$ :

$$
\begin{gathered}
K_{B}(t)=\frac{1}{(4 \pi t)^{m / 2}}\left(\operatorname{vol}^{m}(N)+t^{1 / 2} \frac{\sqrt{\pi}}{2} \operatorname{vol}^{m-1}\left(\Sigma_{\mathrm{ref}} N\right)\right. \\
\left.+t\left(\int_{N} \frac{S}{6}+\frac{\Delta^{N} \psi}{2 \psi}+\frac{1}{4}\left|\frac{d \psi}{\psi}\right|^{2} \mathrm{dvol}+\frac{\pi\left(k^{2}-1\right)}{3 k} \operatorname{vol}^{m-2}\left(\Sigma_{k} N\right)\right)+\mathcal{O}\left(t^{3 / 2}\right)\right) .
\end{gathered}
$$

3.2. Transversally oriented, codimension one Riemannian foliations. Suppose that $(M, \mathcal{F})$ is a transversally oriented, codimension one Riemannian foliation. In this case, the analysis of the basic manifold is unnecessary, because the basic manifold is isometric to the space of leaf closures. For such a foliation, either the closure of every leaf is all of $M$, or the leaves are all compact without holonomy. In the first case, the basic Laplacian is identically zero, so that the trace of the basic heat operator satisfies $K_{B}(t)=1$ for every $t$. In the case of compact leaves, the leaves are the fibers of a Riemannian submersion over a circle. Thus, the basic functions are pullbacks of functions on the circle, and the basic function $v: M \rightarrow \mathbb{R}$ given by $v(x)=($ the volume of the leaf containing $x)$ is smooth on $M$ and likewise smooth in the circle coordinate. If the circle is parametrized to have unit speed by the coordinate $s \in[0, S)$, then the $L^{2}$ inner products on basic functions and basic one-forms are defined by

$$
\begin{aligned}
\langle f, g\rangle & =\int_{0}^{S} f(s) g(s) v(s) d s \\
\langle\alpha(s) d s, \beta(s) d s\rangle & =\int_{0}^{S} \alpha(s) \beta(s) v(s) d s .
\end{aligned}
$$

Note that $S$ is the transverse volume $V_{t r}$ of $(M, \mathcal{F})$, defined as in Theorem 2.3. We then compute that the basic Laplacian on functions is given by

$$
\Delta_{B} f=-\frac{\partial^{2}}{\partial s^{2}} f-\frac{v^{\prime}}{v} \frac{\partial}{\partial s} f
$$

Since the foliation is transversally oriented, we may assume that we have chosen a unit normal vector field $U=\frac{\partial}{\partial s}$. An elementary calculation shows that the total mean curvature $H(s)$ is given by

$$
H(s):=-\ell \int_{L_{s}}\langle\mathbf{H}(x), U(x)\rangle \operatorname{dvol}(x)=\frac{v^{\prime}(s)}{v(s)},
$$


where $\mathbf{H}(x)$ is the mean curvature vector field of the leaf $L_{s}$ corresponding to the coordinate $s$ and $\ell$ is the dimension of each leaf. Recall that $\mathbf{H}$ is defined as follows. Given $x \in L_{s}$, let $\left\{E_{i}\right\}_{i=1}^{\ell}$ be an orthonormal basis of $T_{x} L$. We define

$$
\mathbf{H}(x)=\frac{1}{\ell} \sum_{i=1}^{\ell}\left(\nabla_{E_{i}} E_{i}\right)^{\perp},
$$

where $\perp$ denotes the projection onto the normal space. We denote the mean curvature of $L_{s}$ by $h(x)=\|\mathbf{H}(x)\|$.

In summary, the basic Laplacian on functions $\psi:\left[0, V_{t r}\right] \rightarrow \mathbb{R}$ is given by

$$
\Delta_{B} \psi(s)=-\frac{d^{2}}{d s^{2}} \psi(s)-H(s) \frac{d}{d s} \psi(s) .
$$

The trace of the basic heat kernel may now be computed in the standard way from this operator on the circle. Let an arbitrary point on the circle be denoted by the coordinate 0 , and let $x$ be any other point within $\frac{V_{t r}}{2}$ of 0 . Following the computation in [49, pp. 69-70], we get the following asymptotic expansion of the basic heat kernel $K_{B}(t, x, 0)$ as $t \rightarrow 0$ :

$$
K_{B}(t, x, 0) \sim \frac{e^{-x^{2} / 4 t}}{\sqrt{4 \pi t}}\left(u_{0}(x)+u_{1}(x) t+u_{2}(x) t^{2}+\ldots\right),
$$

where $u_{0}(x)=\frac{1}{R_{-1}(x)}$ and $u_{k+1}(x)$ for $k \geq 0$ is given by

$$
R_{k}(x) u_{k+1}(x)=-\int_{0}^{x} \frac{R_{k}(y)}{y} \Delta_{B} u_{k}(y) d y
$$

where for every $j \geq-1$

$$
\begin{aligned}
R_{j}(x) & =x^{j+1} \exp \left(\frac{1}{2} \int_{0}^{x} H(t) d t\right) \\
& =x^{j+1} \sqrt{\frac{v(0)}{v(x)}}
\end{aligned}
$$

Then (3.5) becomes

$$
x^{k+1} \sqrt{\frac{v(0)}{v(x)}} u_{k+1}(x)=-\int_{0}^{x} y^{k} \frac{v(0)}{v(x)} \Delta_{B} u_{k}(y) d y .
$$

Therefore,

and

$$
u_{0}(x)=\sqrt{\frac{v(x)}{v(0)}}
$$

$$
\begin{aligned}
u_{1}(x) & =\frac{1}{x} \sqrt{\frac{v(x)}{v(0)}} \int_{0}^{x} \sqrt{\frac{v(0)}{v(y)}}\left(\left(\sqrt{\frac{v(y)}{v(0)}}\right)^{\prime \prime}+H(y)\left(\sqrt{\frac{v(y)}{v(0)}}\right)^{\prime}\right) d y \\
& =\frac{1}{4 x} \sqrt{\frac{v(x)}{v(0)}} \int_{0}^{x}\left(\frac{v^{\prime}}{v}\right)^{2}+2 \frac{v^{\prime \prime}}{v} d y \\
& =\frac{1}{4 x} \sqrt{\frac{v(x)}{v(0)}} \int_{0}^{x}\left(2 H^{\prime}(y)+3(H(y))^{2}\right) d y
\end{aligned}
$$


Taking the limit as $x \rightarrow 0$, we obtain

$$
\begin{aligned}
& u_{0}(0)=1, \\
& u_{1}(0)=\frac{1}{4}\left(2 H^{\prime}(0)+3(H(0))^{2}\right) .
\end{aligned}
$$

By realizing that the coordinate 0 was labelled arbitrarily and by integrating the above quantities over the circle, we obtain the following theorem:

Theorem 3.7. Let $(M, \mathcal{F})$ be a transversally oriented Riemannian foliation of codimension one without dense leaves. Then the trace $K_{B}(t)$ of the basic heat operator has the following asymptotic expansion as $t \rightarrow 0$. For any nonnegative integer $J$,

$$
K_{B}(t)=\frac{1}{\sqrt{4 \pi t}}\left(A_{0}+A_{1} t+\ldots+A_{J} t^{J}+\mathcal{O}\left(t^{J+1}\right)\right),
$$

where

$$
\begin{array}{r}
A_{0}=V_{t r}, \\
A_{1}=\frac{3}{4} \ell^{2}\left(\|h\|_{2}\right)^{2},
\end{array}
$$

and the other basic heat invariants may be computed using the recursion formulas and integrations described above. Here, $V_{t r}$ is the transverse volume of the foliation, and $\|h\|_{2}$ is the $L^{2}$ norm of the mean curvature.

Corollary 3.8. Let $(M, \mathcal{F})$ be as in the theorem above. Then the spectrum of the basic Laplacian on functions determines the $L^{2}$ norm of the mean curvature. In particular, the foliation is minimal if and only if $A_{1}=0$ in Theorem 3.7 .

Remark 3.9. The above theorem and corollary may be applied in cases of higher codimension if all of the leaf closures are transversally oriented and have codimension one.

3.3. Codimension one foliations that are not transversally orientable. We now show how the results in the last section need to be modified if $(M, \mathcal{F})$ is not transversally orientable. We will need the results of this section when we consider the case of transversally orientable codimension two foliations whose leaf closures are codimension one and are not necessarily transversally orientable. If $(M, \mathcal{F})$ is a codimension one Riemannian foliation that is not transversally orientable, it has a double cover $(\widetilde{M}, \widetilde{\mathcal{F}})$ that is transversally orientable. Basic functions on $M$ correspond to basic functions on $\widetilde{M}$ that are invariant under the orientationreversing, isometric involution (the deck transformation). Thus the basic Laplacian is a second order operator on a closed interval with Neumann boundary conditions instead of a circle. Part of the analysis from the last section is relevant, so that we obtain the following:

$$
\begin{aligned}
\Delta_{B} \psi(s) & =-\frac{d^{2}}{d s^{2}} \psi(s)-H(s) \frac{d}{d s} \psi(s), \\
\psi^{\prime}(0) & =\psi^{\prime}\left(V_{t r}\right)=0 .
\end{aligned}
$$

The asymptotics of the trace of the associated heat operator is a standard problem. If $\widetilde{K}\left(t, \widetilde{s}_{1}, \widetilde{s}_{2}\right)$ is the lifted heat kernel to the circle, it corresponds to the following 
differential operator on $\left[-V_{t r}, V_{t r}\right]$ with periodic boundary conditions:

$$
L \alpha(\widetilde{s})=-\frac{d^{2}}{d \widetilde{s}^{2}} \alpha(\widetilde{s})-\widetilde{H}(\widetilde{s}) \frac{d}{d \widetilde{s}} \alpha(\widetilde{s}),
$$

where

$$
\widetilde{H}(\widetilde{s})= \begin{cases}H(\widetilde{s}) & \text { if } 0<\widetilde{s}<V_{t r} \\ -H(-\widetilde{s}) & \text { if } \quad-V_{t r}<\widetilde{s}<0\end{cases}
$$

Note that $\widetilde{H}(\widetilde{s})$ is the logarithmic derivative of the volume of the leaf on $(\widetilde{M}, \widetilde{\mathcal{F}})$ and thus extends to be a smooth function on the circle. In particular, this implies that all even derivatives of $\widetilde{H}(\widetilde{s})$ and $H(\widetilde{s})$ at $\widetilde{s}=0$ or $V_{t r}$ are zero. A similar argument shows that the corresponding volume functions $v(\widetilde{s})$ and $\widetilde{v}(\widetilde{s})$ have zero odd derivatives at $\widetilde{s}=0$ or $V_{t r}$. The heat kernel $K\left(t, s_{1}, s_{2}\right)$ for the original boundary value problem (3.7) satisfies

$$
K(t, s, s)=\widetilde{K}(t, s, s)+\widetilde{K}(t, s,-s) .
$$

We have that

$$
\widetilde{K}\left(t, \widetilde{s}_{1}, \widetilde{s}_{2}\right) \sim \frac{e^{-r^{2} / 4 t}}{\sqrt{4 \pi t}}\left(u_{0}\left(\widetilde{s}_{1}, \widetilde{s}_{2}\right)+u_{1}\left(\widetilde{s}_{1}, \widetilde{s}_{2}\right) t+\ldots\right),
$$

where $r=\operatorname{dist}\left(\widetilde{s}_{1}, \widetilde{s}_{2}\right)$ and the functions $u_{j}$ are explicitly computable from the differential equation (3.3). The trace is computed by the integral

$$
\begin{aligned}
K_{B}(t) & =\int_{0}^{V_{t r}} K(t, s, s) d s \\
& =\int_{0}^{V_{t r}} \widetilde{K}(t, s, s)+\widetilde{K}(t, s,-s) d s \\
& =\frac{1}{2} \int_{-V_{t r}}^{V_{t r}} \widetilde{K}(t, s, s)+\widetilde{K}(t, s,-s) d s \\
& \sim \frac{1}{\sqrt{4 \pi t}}\left(A_{0}+B_{0} t^{1 / 2}+A_{1} t+B_{1} t^{3 / 2}+\ldots\right),
\end{aligned}
$$

where $A_{j}$ is defined as in the oriented case, and $B_{j}$ depends on the derivatives of $u_{j}$ evaluated at $(0,0)$ and $\left(V_{t r}, V_{t r}\right)$. The first nontrivial coefficients in the formula are

$$
\begin{aligned}
A_{0}= & \frac{1}{2} \int_{-V_{t r}}^{V_{t r}} u_{0}(\widetilde{s}, \widetilde{s}) d \widetilde{s} \\
B_{0}= & \frac{\sqrt{\pi}}{2}\left(u_{0}(0,0)+u_{0}\left(V_{t r}, V_{t r}\right)\right) \\
A_{1}= & \frac{1}{2} \int_{-V_{t r}}^{V_{t r}} u_{1}(\widetilde{s}, \widetilde{s}) d \widetilde{s} \\
B_{1}= & \frac{\sqrt{\pi}}{2}\left(u_{1}(0,0)+u_{1}\left(V_{t r}, V_{t r}\right)\right) \\
& +\frac{\sqrt{\pi}}{8}\left(\partial_{1} \partial_{1}-2 \partial_{1} \partial_{2}+\partial_{2} \partial_{2}\right) u_{0}(0,0) \\
& +\frac{\sqrt{\pi}}{8}\left(\partial_{1} \partial_{1}-2 \partial_{1} \partial_{2}+\partial_{2} \partial_{2}\right) u_{0}\left(V_{t r}, V_{t r}\right) .
\end{aligned}
$$


From the calculations in the transversally oriented case, we have

$$
\begin{aligned}
& u_{0}\left(s_{1}, s_{2}\right)=\sqrt{\frac{v\left(s_{1}\right)}{v\left(s_{2}\right)}}, \\
& u_{1}\left(s_{1}, s_{2}\right)=\frac{1}{4\left(s_{1}-s_{2}\right)} \sqrt{\frac{v\left(s_{1}\right)}{v\left(s_{2}\right)}} \int_{s_{2}}^{s_{1}}\left(2 \widetilde{H}^{\prime}(y)+3(\widetilde{H}(y))^{2}\right) d y
\end{aligned}
$$

which after some calculation implies that

$$
\begin{aligned}
u_{0}(\widetilde{s}, \widetilde{s}) & =1, \\
u_{1}(\widetilde{s}, \widetilde{s}) & =\frac{1}{4}\left(2 \widetilde{H}^{\prime}(\widetilde{s})+3(\widetilde{H}(\widetilde{s}))^{2}\right), \\
\left(\partial_{1} \partial_{1}-2 \partial_{1} \partial_{2}+\partial_{2} \partial_{2}\right) u_{0}(0,0) & =0, \\
\left(\partial_{1} \partial_{1}-2 \partial_{1} \partial_{2}+\partial_{2} \partial_{2}\right) u_{0}\left(V_{t r}, V_{t r}\right) & =0 .
\end{aligned}
$$

These equations imply that

$$
\begin{aligned}
A_{0} & =V_{t r}, \\
B_{0} & =\sqrt{\pi} \\
A_{1} & =\frac{1}{8} \int_{-V_{t r}}^{V_{t r}}\left(2 \widetilde{H}^{\prime}(\widetilde{s})+3(\widetilde{H}(\widetilde{s}))^{2}\right) d \widetilde{s} \\
& =\frac{3}{8} \int_{-V_{t r}}^{V_{t r}}(\widetilde{H}(\widetilde{s}))^{2} d \widetilde{s}=\frac{3}{4} \int_{0}^{V_{t r}}(H(s))^{2} d s, \\
B_{1} & =\frac{\sqrt{\pi}}{8}\left(2 \widetilde{H}^{\prime}(0)+3(\widetilde{H}(0))^{2}+2 \widetilde{H}^{\prime}\left(V_{t r}\right)+3\left(\widetilde{H}\left(V_{t r}\right)\right)^{2}\right) \\
& =\frac{\sqrt{\pi}}{4}\left(\widetilde{H}^{\prime}(0)+\widetilde{H}^{\prime}\left(V_{t r}\right)\right)=\frac{\sqrt{\pi}}{4}\left(H^{\prime}(0)+H^{\prime}\left(V_{t r}\right)\right) .
\end{aligned}
$$

In summary, we have the following theorem.

Theorem 3.10. Let $(M, \mathcal{F})$ be a Riemannian foliation of codimension one such that the leaves are not dense and the foliation is not transversally orientable. Then the trace $K_{B}(t)$ of the basic heat operator has the following asymptotic expansion as $t \rightarrow 0$ :

$$
K_{B}(t)=\frac{1}{\sqrt{4 \pi t}}\left(A_{0}+B_{0} t^{1 / 2}+A_{1} t+B_{1} t^{3 / 2}+\ldots\right),
$$

where

$$
\begin{aligned}
A_{0} & =V_{t r}, \\
B_{0} & =\sqrt{\pi} \\
A_{1} & =\frac{3}{4} \ell^{2}\left(\|h\|_{2}\right)^{2}, \\
B_{1} & =\frac{\sqrt{\pi}}{4}\left(F(0)+F\left(V_{t r}\right)\right)
\end{aligned}
$$

and the other basic heat invariants may be computed using the techniques described above. Here, $V_{t r}$ is the transverse volume of the foliation, $\|h\|_{2}$ is the $L^{2}$ norm of the mean curvature, and $F(0)+F\left(V_{t r}\right)$ is the sum of the second normal derivatives 
of the logarithm of leaf volume, evaluated at the two leaves with $\mathbb{Z}_{2}$ holonomy (this quantity is independent of the choice of normal at any point of these leaves).

Corollary 3.11. Let $(M, \mathcal{F})$ be a codimension one Riemannian foliation. Then the spectrum of the basic Laplacian on functions determines whether or not the leaves are dense. If the leaves are not dense, the spectrum also determines whether or not the foliation is transversally orientable, the $L^{2}$ norm of the mean curvature, and the average of the second normal derivatives of the logarithm of leaf volume at the two leaves with $\mathbb{Z}_{2}$ holonomy in the nonorientable case. In particular, the foliation is minimal if and only if the coefficient $A_{1}=0$.

Remark 3.12. The above theorem and corollary may be applied in cases of higher codimension if all of the leaf closures have codimension one.

3.4. The general case. We now prove some results that will be applied to the codimension two case in Section 3.5. These results are completely general and may be used to compute the asymptotic expansion in special cases of arbitrary codimension. We first review results that will be used in our computations. Recall that $\widetilde{K}(t, w, v):=\widetilde{K_{W}}(t, w, v)$ is the heat kernel corresponding to the operator $\widetilde{\Delta_{W}}$ defined in (2.2), so that if $\varepsilon>0$ is sufficiently small, $\operatorname{dist}(w, v)>\varepsilon$ implies that

$$
\widetilde{K}(t, w, v)=\mathcal{O}\left(e^{-c / t}\right)
$$

as $t \rightarrow 0$, for some constant $c$ (see, for example, [27]). As a consequence, the asymptotics of the integral in Proposition 2.1 over $G \times W$ are the same as the asymptotics of the integral over $U$, where $U$ is any arbitrarily small neighborhood of the compact subset $\{(g, w) \in G \times W \mid w g=w\}$, up to an error term of the form $O\left(e^{-c / t}\right)$.

We will now decompose $W$ into pieces and use this decomposition to partition a neighborhood of $\{(g, w) \in G \times W \mid w g=w\}$. Given an orbit $X$ of $G$ and $w \in X, X$ is naturally diffeomorphic to $G / H_{w}$, where $H_{w}=\{g \in G \mid w g=w\}$ is the (closed) isotropy subgroup. As we mentioned before, $H_{w}$ is isomorphic to the structure group corresponding to the principal bundle $\pi: \rho^{-1}(w) \rightarrow \bar{L}$, where $\bar{L}$ is the leaf closure $\pi\left(\rho^{-1}(w)\right)$ in $M$. Given a subgroup $H$ of $G$, let $[H]$ denote the conjugacy class of $H$. The isotropy type of the orbit $X$ is defined to be the conjugacy class $\left[H_{w}\right]$, which is well-defined independent of $w \in X$. There are a finite number of isotropy types of orbits in $W$ (see [7, p. 173]). We define the usual partial ordering (see [7, p. 42]) on the isotropy types by declaring that

$$
[H] \leq[K] \Longleftrightarrow H \text { is conjugate to a subgroup of } K \text {. }
$$

Let $\left\{\left[H_{i}\right]: i=1, \ldots, r\right\}$ be the set of isotropy types occurring in $W$, arranged so that

$$
\left[H_{i}\right] \leq\left[H_{j}\right] \Longrightarrow i \geq j
$$

(see [30, p. 51]). Let $W([H])$ denote the union of orbits of isotropy type $[H]$ in $W$. The set $W\left(\left[H_{i}\right]\right)$ is in general a $G$-invariant submanifold of $W$ (see [30, p. 202]). Also, $W\left(\left[H_{1}\right]\right)$ is closed, and $W\left(\left[H_{r}\right]\right)$ is open and dense in $W([30$, p. 50, 216]). Thus, $W$ is the disjoint union of the submanifolds $W\left(\left[H_{i}\right]\right)$ for $1 \leq i \leq r$. 
Now, given a proper, $G$-invariant submanifold $S$ of $W$ and $\varepsilon>0$, let $T_{\varepsilon}(S)$ denote the union of the images of the exponential map at $s$ for $s \in S$ restricted to the ball of radius $\varepsilon$ in the normal bundle at $S$. It follows that $T_{\varepsilon}(S)$ is also $G$-invariant. We now decompose $W$ as a disjoint union of sets $W_{1}, \ldots, W_{r}$. If there is only one isotropy type on $W$, then $r=1$, and we let $W_{1}=W$. Otherwise, let $W_{1}=T_{\varepsilon}\left(W\left(\left[H_{1}\right]\right)\right)$. For $1<j \leq r-1$, let

$$
W_{j}=T_{\varepsilon}\left(W\left(\left[H_{j}\right]\right)\right) \backslash \bigcup_{i=1}^{j-1} W_{i} .
$$

Let

$$
W_{r}=W \backslash \bigcup_{i=1}^{r-1} W_{i}
$$

Clearly, $\varepsilon>0$ must be chosen sufficiently small in order for the following lemma to be valid. We in addition insist that $\varepsilon$ be chosen sufficiently small so that the asymptotic expansion for $\widetilde{K_{W}}(t, x, y)$ is valid if the distance from $x$ to $y$ is less than $\varepsilon$. The following facts about this decomposition are contained in [30, pp. 203ff.]:

Lemma 3.13. With $W, W_{i}$ defined as above, we have, for every $i \in\{1, \ldots, r\}$ :

(1) $W=\bigcup_{i=1}^{r} W_{i}$.

(2) $W_{i}$ is a union of $G$-orbits.

(3) The closure of $W_{i}$ is a compact $G$-manifold with corners.

(4) If $\left[H_{j}\right]$ is the isotropy type of an orbit in $W_{i}$, then $j \geq i$.

(5) The distance between the submanifold $W\left(\left[H_{j}\right]\right)$ and $W_{i}$ for $j<i$ is at least $\varepsilon$.

Remark 3.14. The lemma above remains true if at each stage $T_{\varepsilon},\left(W\left(\left[H_{j}\right]\right)\right)$ is replaced by any sufficiently small open neighborhood of $W\left(H_{j}\right)$ that contains $T_{\varepsilon}\left(W\left(\left[H_{j}\right]\right)\right)$, that is a union of $G$-orbits, and whose closure is a manifold with corners.

Therefore, by Proposition 2.1, the trace of the basic heat kernel is given by

$$
K_{B}(t)=\sum_{i=1}^{r} \int_{W_{i}} \int_{G} \widetilde{K}(t, w, w g) \chi(g) \operatorname{dvol}_{W}(w) .
$$

Let $H_{j}$ be the isotropy subgroup of $w \in W\left(\left[H_{j}\right]\right)$, and let $\gamma$ be a geodesic orthogonal to $W\left(\left[H_{j}\right]\right)$ through $w$. This situation occurs exactly when this geodesic is orthogonal both to the fixed point set $W^{H_{j}}$ of $H_{j}$ and to the orbit $w G$ of $G$ containing $w$. For any $h \in H_{j}$, right multiplication by $h$ maps geodesics orthogonal to $W^{H_{j}}$ through $w$ to themselves and likewise maps geodesics orthogonal to $w G$ through $w$ to themselves. Thus, the group $H_{j}$ acts orthogonally on the normal space to $w \in W\left(\left[H_{j}\right]\right)$ by the differential of right multiplication. Observe in addition that there are no fixed points for this action; that is, there is no element of the normal space that is fixed by every $h \in H_{j}$. If $G=S O(q)$ acts by orientation-preserving isometries, then $H_{j}$ acts on the normal space in the same way. Since $H_{j}$ acts without fixed points, the codimension of $W\left(\left[H_{j}\right]\right)$ is at least two in the orientationpreserving case. 
On the other hand, if the transformation group $G$ in question is abelian and acts by orientation-preserving isometries, then the representation theory of abelian groups implies that the representation space must be even-dimensional (see [8, pp. 107-110]). In this case, we would then conclude that the codimension of each $W\left(\left[H_{j}\right]\right)$ is even. We mention this for the following reason. Since $\widetilde{\Delta_{W}}$ commutes with the $S O(q)$ action on the basic manifold $W$, the integrand $\widetilde{K}(t, w, w g)$ in Proposition 2.1 is a class function, so that Weyl's integration formula may be used to rewrite the integral as an integral over a maximal torus $T$ of $S O(q)$ (see [8, pp. 163]). Formula (3.8) becomes

$$
K_{B}(t)=\sum_{i=1}^{r} \int_{W_{i}} \int_{T} \tilde{K}(t, w, w g) \eta(g) \operatorname{dvol}_{W}(w),
$$

where $\eta(g)$ is the volume form on $T$ multiplied by a bounded function of $g \in T$. In the above expression, we may take $W_{i}$ to be those constructed using $G=T$. Therefore, each $H_{j}$ is a subgroup of the torus, and the codimension of each $W\left(\left[H_{i}\right]\right)$ is even in the orientation-preserving case, as has been explained previously.

As $t \rightarrow 0$, we need only evaluate the asymptotics of the integrals in (3.9) on an arbitrarily small neighborhood of the set $\{(g, w) \in T \times W \mid w g=w\}$. By the construction of $W_{i}$, the integral over $T$ may be replaced by an integral over a small neighborhood of $H_{i}$ in $T$. This neighborhood may be described as $N_{\varepsilon^{\prime}}\left(H_{i}\right)=$ $\left\{g h \mid h \in H_{i}, g \in B_{\varepsilon^{\prime}}\right\}$, where $B_{\varepsilon^{\prime}}$ is a ball of radius $\varepsilon^{\prime}$ centered at the identity in $\exp _{e} H_{i}^{\perp}$. Here, $H_{i}^{\perp}$ is the normal space to $H_{i} \subset T$ at the identity $e$, and $\exp _{e}$ is the exponential map $\exp _{e}: \mathfrak{t} \rightarrow T$. We have

$$
K_{B}(t)=\sum_{i=1}^{r} \int_{W_{i}} \int_{B_{\varepsilon^{\prime}} \times H_{i}} \widetilde{K}(t, w, w g h) \eta^{\prime}(g, h) \operatorname{dvol}_{W}(w)+\mathcal{O}\left(e^{-c / t}\right) .
$$

We can now make this integral over the torus explicit. A maximal torus of $S O(q)$ has dimension $\left[\frac{q}{2}\right]$, and we define $T$ in the following way. Let $\Theta=\left(\theta_{1}, \ldots, \theta_{m}\right) \in$ $(-\pi, \pi]^{m}$. If $q=2 m$, let

$$
T=\left\{M(\Theta) \mid \theta_{j} \in(-\pi, \pi] \text { for every } j\right\},
$$

where

$$
M(\Theta)=\left(\begin{array}{ccccc}
\cos \theta_{1} & \sin \theta_{1} & \ldots & 0 & 0 \\
-\sin \theta_{1} & \cos \theta_{1} & \ldots & 0 & 0 \\
\vdots & \vdots & \ddots & \vdots & \vdots \\
0 & 0 & \ldots & \cos \theta_{m} & \sin \theta_{m} \\
0 & 0 & \ldots & -\sin \theta_{m} & \cos \theta_{m}
\end{array}\right)
$$

If $q=2 m+1, T$ is defined similarly with

$$
M(\Theta)=\left(\begin{array}{cccccc}
\cos \theta_{1} & \sin \theta_{1} & \ldots & 0 & 0 & 0 \\
-\sin \theta_{1} & \cos \theta_{1} & \ldots & 0 & 0 & 0 \\
\vdots & \vdots & \ddots & \vdots & \vdots & \vdots \\
0 & 0 & \ldots & \cos \theta_{m} & \sin \theta_{m} & 0 \\
0 & 0 & \ldots & -\sin \theta_{m} & \cos \theta_{m} & 0 \\
0 & 0 & \ldots & 0 & 0 & 1
\end{array}\right) .
$$


Then we have the following formulas ([8, pp. 171, 219-221]) for the form $\eta$ in formula (3.9):

$$
\eta(\Theta)=\frac{1}{m ! 2^{m-1}} \prod\left(1-e^{ \pm i \theta_{k} \pm i \theta_{l}}\right) \cdot \frac{d \Theta}{(2 \pi)^{m}}
$$

if $q=2 m$, and

$$
\eta(\Theta)=\frac{1}{m ! 2^{m}} \prod\left(1-e^{ \pm i \theta_{k} \pm i \theta_{l}}\right) \prod\left(1-e^{ \pm i \theta_{j}}\right) \cdot \frac{d \Theta}{(2 \pi)^{m}}
$$

if $q=2 m+1$. Simplifying, we get

$$
\eta(\Theta)=\frac{2^{m^{2}-3 m+1}}{m ! \pi^{m}}\left(\prod_{1 \leq k<l \leq m}\left(\cos \theta_{k}-\cos \theta_{l}\right)^{2}\right) d \Theta
$$

if $q=2 m$, and

$$
\eta(\Theta)=\frac{2^{m^{2}-2 m}}{m ! \pi^{m}}\left(\prod_{1 \leq k<l \leq m}\left(\cos \theta_{k}-\cos \theta_{l}\right)^{2}\right)\left(\prod_{1 \leq j \leq m}\left(1-\cos \theta_{j}\right)\right) d \Theta
$$

if $q=2 m+1$. Equation (3.9) becomes

$$
K_{B}(t)=\sum_{i=1}^{r} \int_{W_{i}} \int_{(-\pi, \pi)^{m}} \widetilde{K}(t, w, w M(\Theta)) \eta(\Theta) \operatorname{dvol}_{W}(w),
$$

using the appropriate choice of $\eta(\Theta)$ above. The explicit description of $\eta(\Theta)$ may be used to make equation (3.10) more explicit as well, but additional calculations and a choice of coordinates on $\exp _{e} H_{i}^{\perp}$ are necessary.

3.5. Codimension two Riemannian foliations. We now explicitly derive the coefficients in the asymptotic expansion of the trace of the basic heat operator on transversally oriented Riemannian foliations of codimension two. We have the following possibilities:

(1) (The trivial case.) The closure of every leaf of $(M, \mathcal{F})$ is the manifold $M$. In this case, the basic functions are constants, and the basic heat operator is the identity. Therefore, the trace $K_{B}(t)$ of the basic heat operator satisfies $K_{B}(t)=1$ for every $t$.

(2) Every leaf of $(M, \mathcal{F})$ is closed. Then the results of Section 3.1 apply. The basic manifold $W$ is three-dimensional. Given $w \in W$, the $S O(2)$ orbit $X$ of $w$ is a circle.

(3) Each leaf closure of $(M, \mathcal{F})$ has codimension one. If $\overline{\mathcal{F}}$ denotes the collection of leaf closures of $(M, \mathcal{F}),(M, \overline{\mathcal{F}})$ is a Riemannian foliation of codimension one. Observe that the basic functions, $L^{2}$ inner products, and basic Laplacians for $(M, \mathcal{F})$ and $(M, \overline{\mathcal{F}})$ are the same, so that we have reduced to the nontrivial codimension one cases. If the leaf closure foliation is transversally orientable, see Section 3.2. If the leaf closure foliation is not transversally orientable, see Section 3.3 .

(4) The leaf closures of $(M, \mathcal{F})$ have minimum codimension one, but some leaf closures have codimension two. This situation is the most interesting case that arises. At least one of the orbits has finite isotropy. Thus, the basic manifold $W$ is a closed two-manifold with an $S O(2)$ action whose 
orbits have two different dimensions. The circular orbits correspond to the codimension one leaf closures, and the (isolated) fixed points correspond to the codimension two leaf closures. Because the group action yields a vector field on $W$ that has index 1 at each fixed point, the Euler characteristic of $W$ must be a positive integer. Therefore, $W$ is a sphere or a projective plane; for simplicity we consider only the case where $W=S^{2}$. The metric is a function of the height (orbit) multiplied by the standard metric on the sphere. The space of leaf closures of $(M, \mathcal{F})$ is a closed interval. This case could be considered as a one-dimensional problem (as in Section 3.2), but the analysis is quite difficult because the mean curvature of the leaf closures goes to infinity at the leaves with infinite holonomy. Instead, we use the approach of Section 3.4. The circular orbits have orbit type $(\{e\})$, and the fixed points have orbit type $(S O(2))$. Since $(\{e\}) \leq(S O(2))$, we can decompose $W=W_{1} \cup W_{2}$ as in Lemma 3.13, where $W_{1}$ is the union of two metric $\varepsilon$-disks centered at the fixed points and $W_{2}=W \backslash W_{1}$. The maximal torus of $S O(2)$ is itself, and equation (3.11) shows that the trace of the basic heat operator is

$$
\begin{aligned}
K_{B}(t) & =\int_{W_{1}} \int_{-\pi}^{\pi} \widetilde{K}(t, w, w M(\theta)) \frac{d \theta}{2 \pi} \operatorname{dvol}_{W}(w) \\
& +\int_{W_{2}} \int_{-\pi}^{\pi} \widetilde{K}(t, w, w M(\theta)) \frac{d \theta}{2 \pi} \operatorname{dvol}_{W}(w),
\end{aligned}
$$

where $M(\theta)=\left(\begin{array}{cc}\cos \theta & -\sin \theta \\ \sin \theta & \cos \theta\end{array}\right)$

We now state a result from [47:

Theorem 3.15 (in [47]). Let $\psi: M \rightarrow \mathbb{R}$ be the smooth basic function defined by setting $\psi(x)$ equal to the volume of any leaf closure of $\widehat{M}$ which intersects the fiber $\pi^{-1}(x)$. Let $q_{x}$ denote the codimension of the leaf closure $\bar{L}_{x}$ containing $x$ in $M$. Then, as $t \rightarrow 0$, we have the following asymptotic expansion for any positive integer $k$ :

$$
K_{B}(t, x, x)=\frac{1}{(4 \pi t)^{q_{x} / 2}}\left(a_{0}(x)+a_{1}(x) t+\ldots+a_{k}(x) t^{k}+\mathcal{O}\left(t^{k+1}\right)\right),
$$

where

$$
a_{i}(x)=\sum \frac{2^{l} K_{m_{1} \ldots m_{l}}^{j l}(x) I_{m_{1} \ldots m_{l}}^{Q l}}{\operatorname{Vol}\left(\bar{L}_{x}\right) \pi^{Q / 2}}
$$

The constants $I_{m_{1} \ldots m_{l}}^{Q l}$ are defined by integrals, and the functions $K_{m_{1} \ldots m_{l}}^{j l}(x)$ are determined by the methods in [47]. The integer $Q$ is the codimension of the intersection of the leaf closure containing $\hat{x}$ with $\pi^{-1}(x)$ in $\pi^{-1}(x)$. The functions $a_{i}(x)$ are determined by the local geometry of the foliation at $x \in M$ and by $\psi(x)$. In particular, $a_{0}(x)=\frac{1}{\operatorname{Vol}\left(\overline{L_{x}}\right)}$ and

$$
\begin{gathered}
a_{1}(x)=\frac{1}{\operatorname{Vol}\left(\bar{L}_{x}\right)}\left(\frac{S^{W}(\rho(\hat{x}))}{6}+\frac{\Delta_{B} \psi}{2 \psi}(x)+\frac{3(d \psi, d \psi)}{4 \psi^{2}}(x)\right. \\
\left.+\frac{1}{6} C_{X}^{12} \operatorname{Ric}^{W}(\rho(\hat{x}))+\frac{1}{12} C^{34} C^{12} T(\rho(\hat{x}))+\frac{1}{6} C^{24} C^{13} T(\rho(\hat{x}))-\frac{S^{X}(\rho(\hat{x}))}{3}\right) .
\end{gathered}
$$


The curvature terms are evaluated at any $\hat{x} \in \pi^{-1}(x)$ and are independent of that choice. In the above equation, $S^{X}$ and $S^{W}$ denote the scalar curvatures of $X=$ $\rho\left(\pi^{-1}(x)\right)$ and $W$, respectively, $\operatorname{Ric}^{W}$ is the Ricci curvature tensor on $W, C^{a b}$ denotes tensor contraction in the $a^{\text {th }}$ and $b^{\text {th }}$ slots, and the subscript $X$ means that the contraction is taken over the tangent space to $X$ at $\rho(\hat{x})$. The $(0,4)$-tensor $T$ on $X$ is defined by

$$
\begin{aligned}
T(V, W, Y, Z) & =\left\langle\nabla_{V}^{\perp} W, \nabla_{Y}^{\perp} Z\right\rangle^{\perp} \text { and } \\
T_{k p r t} & =T\left(\partial_{k}, \partial_{p}, \partial_{r}, \partial_{t}\right) .
\end{aligned}
$$

The symbol $\perp$ refers to the orthogonal complement of $T_{\rho(\hat{x})} X$.

The asymptotics of the second term in (3.12) can be found by integrating the asymptotics in Theorem 3.15 , because the coefficients $a_{j}(x)$ and error estimates are bounded on the set $\left\{x \in M \mid \rho\left(\pi^{-1}(x)\right) \subset W_{2}\right\}$. We note that all of these coefficient functions arise from such an integral on $W$. Explicitly, for $w \in W_{2}$,

$$
\begin{aligned}
\int_{-\pi}^{\pi} \tilde{K}(t, w, w M(\theta)) \frac{d \theta}{2 \pi} & =\frac{1}{\sqrt{4 \pi t}}\left(a_{0}(w)+a_{1}(w) t\right. \\
& \left.+\ldots+a_{k}(w) t^{k}+\mathcal{O}\left(t^{k+1}\right)\right)
\end{aligned}
$$

where

$$
a_{0}(w)=\frac{\phi(w)}{\operatorname{Vol}\left(\pi\left(\rho^{-1}(w)\right)\right)}=\frac{1}{\operatorname{Vol}\left(X_{w}\right)},
$$

letting $\phi(w)$ be the volume of the leaf closure $\rho^{-1}(w)$ in $\widehat{M}$ and letting $X=X_{w}$ be the orbit of $w$ in $W$. Observe that we have also used (2.1) to convert the integral over $M$ to an integral over $W$. Similarly, $a_{1}(w)$ is obtainable from the expression for $a_{1}$ in the theorem. Since the orbits are one-dimensional and $W$ is two-dimensional,

$$
\begin{aligned}
S^{X}(w) & =0, \\
S^{W}(w) & =2 K(w), \\
C_{X}^{12} \operatorname{Ric}^{W}(w) & =\operatorname{Ric}^{W}\left(\sigma_{w}, \sigma_{w}\right)=K(w), \\
C^{24} C^{13} T(w) & =C^{34} C^{12} T(w)=\|\mathbf{H}(w)\|^{2}=\kappa(w)^{2},
\end{aligned}
$$

where $K(w)$ is the Gauss curvature of the basic manifold at $w$ and $\kappa(w)$ is the geodesic curvature of the orbit at $w$. Also, if $x \in \pi\left(\rho^{-1}(w)\right)$ and $\widehat{x} \in \pi^{-1}(x)$,

$$
\begin{aligned}
\frac{\Delta_{B} \psi}{2 \psi}(x)+\frac{3(d \psi, d \psi)}{4 \psi^{2}}(x) & =\frac{\pi^{*} \Delta_{B} \psi}{2 \pi^{*} \psi}(\widehat{x})+\frac{3\left(\pi^{*} d \psi, \pi^{*} d \psi\right)}{4 \pi^{*} \psi^{2}}(\widehat{x}) \\
& =\frac{\widehat{\Delta_{B}} \pi^{*} \psi}{2 \pi^{*} \psi}(\widehat{x})+\frac{3\left(d \pi^{*} \psi, d \pi^{*} \psi\right)}{4 \pi^{*} \psi^{2}}(\widehat{x}) \\
& =\frac{\widehat{\Delta_{B}} \rho^{*} \phi}{2 \rho^{*} \phi}(\widehat{x})+\frac{3\left(d \rho^{*} \phi, d \rho^{*} \phi\right)}{4 \rho^{*} \phi^{2}}(\widehat{x}) \\
& =\frac{\rho^{*} \widehat{\Delta_{W}} \phi}{2 \rho^{*} \phi}(\widehat{x})+\frac{3\left(d \rho^{*} \phi, d \rho^{*} \phi\right)}{4 \rho^{*} \phi^{2}}(\widehat{x}) \\
& =\frac{\Delta_{W} \phi}{2 \phi}(w)+\frac{(d \phi, d \phi)}{4 \phi^{2}}(w),
\end{aligned}
$$


using the definition of $\widetilde{\Delta_{W}}$ (see Section 22). Therefore, we now obtain

$$
a_{1}(w)=\frac{1}{\operatorname{Vol}\left(X_{w}\right)}\left(\frac{1}{2} K(w)+\frac{1}{4} \kappa(w)^{2}+\frac{\Delta_{W} \phi}{2 \phi}(w)+\frac{(d \phi, d \phi)}{4 \phi^{2}}(w)\right)
$$

and

$$
\begin{aligned}
\int_{W_{2}} \int_{-\pi}^{\pi} \widetilde{K}(t, w, w M(\theta)) \frac{d \theta}{2 \pi} \operatorname{dvol}_{W}(w) \\
\quad=\frac{1}{\sqrt{4 \pi t}} \int_{W_{2}}\left(a_{0}(w)+a_{1}(w) t+O\left(t^{2}\right)\right) \operatorname{dvol}_{W}(w) .
\end{aligned}
$$

In the expression above, note that we could take the limit as $\varepsilon \rightarrow 0$ (that is, as $\left.W_{2} \rightarrow W\right)$ in every term except the one involving the geodesic curvature $\kappa(w)$.

(1) Next, we determine the asymptotics of the integral over $W_{1}$ in (3.12). Recall that $W_{1}$ is the disjoint union of two metric $\varepsilon$-disks $D_{1}$ and $D_{2}$ surrounding the singular orbits. Choosing geodesic polar coordinates $(r, \gamma)$ around one of the singular orbits, let $C(r)$ denote the length of the orbit at radius $r$. Then $C(r)=$ $2 \pi r\left(1+\mathcal{O}\left(r^{2}\right)\right)$. The metric on the basic manifold is

$$
\left(g_{i j}(r, \gamma)\right)=\left(\begin{array}{cc}
1 & 0 \\
0 & \frac{C(r)^{2}}{4 \pi^{2}}
\end{array}\right)
$$

where $g_{11}(r, \gamma)=\left\langle\partial_{r}, \partial_{r}\right\rangle$, and so on. The integral over $D_{1}$ is

$$
\begin{aligned}
\int_{D_{1}} \int_{-\pi}^{\pi} & \widetilde{K}(t, w, w M(\theta)) \frac{d \theta}{2 \pi} \operatorname{dvol}_{W}(w) \\
= & \int_{r=0}^{\varepsilon} \int_{\gamma=-\pi}^{\pi} \int_{-\pi}^{\pi} \widetilde{K}(t,(r, \gamma),(r, \gamma) M(\theta)) \frac{d \theta}{2 \pi} d \gamma \frac{C(r)}{2 \pi} d r \\
= & \int_{r=0}^{\varepsilon} \int_{0}^{\pi} \widetilde{K}(t,(r, 0),(r, 0) M(\theta)) d \theta \frac{C(r)}{\pi} d r
\end{aligned}
$$

since the integrand is independent of $\gamma$ because of the isometric action of $M(\theta)$. The Minakshisundaram-Pleijel expansion of $\widetilde{K}(t,(r, 0),(r, 0) M(\theta))$ is of the form

$$
\begin{aligned}
& \widetilde{K}(t,(r, 0),(r, 0) M(\theta)) \\
= & \frac{1}{4 \pi t} e^{-D^{2}(r, \theta) / 4 t}\left(u_{0}(r, \theta)+u_{1}(r, \theta) t+u_{2}(r, \theta) t^{2}+\mathcal{O}\left(t^{3}\right)\right),
\end{aligned}
$$

where $D(r, \theta)$ is geodesic distance between $(r, 0)$ and $(r, 0) M(\theta)$. Each $u_{j}(r, \theta)$ is a smooth, bounded function on the $\varepsilon$-disk. Next, observe that $D(r, 0)=0$, $D(r, \pm \pi)=2 r$, and $D^{2}(r, \theta)=r^{2}\left((1-\cos \theta)^{2}+\sin ^{2} \theta\right)+\mathcal{O}\left(r^{3}\right)=2 r^{2}(1-\cos \theta)$ $+\mathcal{O}\left(r^{3}\right)$ for small $r$. Using properties of the exponential map, one could easily show that $D(r, \theta)$ increases in $\theta$ on $[0, \pi]$. Hence, after changing coordinates in $\theta$ 
(affecting the $u_{j}(r, \theta)$ by $O(r)$ ), we have that

$$
\begin{aligned}
& \int_{D_{1}} \int_{-\pi}^{\pi} \widetilde{K}(t, w, w M(\theta)) \frac{d \theta}{2 \pi} \operatorname{dvol}_{W}(w) \\
= & \int_{r=0}^{\varepsilon} \int_{0}^{\pi} \widetilde{K}(t,(r, 0),(r, 0) M(\theta)) d \theta \frac{C(r)}{\pi} d r \\
= & \frac{1}{4 \pi t} \int_{r=0}^{\varepsilon} \int_{0}^{\pi} e^{-\frac{r^{2}}{2 t}(1-\cos \theta)}\left(\bar{u}_{0}(r, \theta)+\bar{u}_{1}(r, \theta) t+\mathcal{O}\left(t^{2}\right)\right) d \theta \frac{C(r)}{\pi} d r \\
(3.13)= & \frac{1}{4 \pi t} \int_{r=0}^{\varepsilon} \int_{0}^{\pi} e^{-\frac{r^{2}}{2 t}(1-\cos \theta)}\left(b_{0}(r, \theta)+b_{1}(r, \theta) t+\mathcal{O}\left(t^{2}\right)\right) d \theta 2 r d r,
\end{aligned}
$$

where by symmetry we may assume that $\left.\frac{\partial^{j}}{\partial r^{j}} b_{m}(r, \theta)\right|_{r=0}=0$ for $j$ odd.

We now compute the asymptotics of some integrals that will be used in our calculation. Let

$$
I_{1}(t, \varepsilon)=\int_{r=0}^{\varepsilon} \int_{0}^{\pi} e^{-\frac{r^{2}}{2 t}(1-\cos \theta)} d \theta 2 r d r .
$$

By Tonelli's theorem we change the order of integration, and we then integrate to get

$$
I_{1}(t, \varepsilon)=2 \varepsilon^{2} s \int_{0}^{\pi} \frac{\left(1-e^{-\frac{1}{2 s}(1-\cos \theta)}\right)}{(1-\cos \theta)} d \theta,
$$

letting $s=\frac{t}{\varepsilon^{2}}$. By changing variables by $\cos \theta=\frac{x-s}{x+s}=1-\frac{2 s}{x+s}$, we obtain

$$
\begin{aligned}
I_{1}(t, \varepsilon) & =2 \varepsilon^{2} s \int_{0}^{\infty} \frac{\left(1-e^{-\frac{1}{x+s}}\right)}{\frac{2 s}{x+s}} \frac{\sqrt{s} d x}{\sqrt{x}(x+s)} \\
& =\varepsilon^{2} \sqrt{s} \int_{0}^{\infty} \frac{\left(1-e^{-\frac{1}{x+s}}\right)}{\sqrt{x}} d x \\
& =\varepsilon^{2} \sqrt{s} F(s) .
\end{aligned}
$$

Using a Lebesgue convergence theorem argument, it can be shown that the integral $F(s)$ is smooth in $s$ and can be differentiated under the integral sign. In fact, it can be shown that

$$
F(s)=\int_{0}^{\infty} \frac{\left(1-e^{-\frac{1}{x+s}}\right)}{\sqrt{x}} d x=(2 \sqrt{\pi})+\left(-\frac{1}{2} \sqrt{\pi}\right) s+\mathcal{O}\left(s^{2}\right)
$$

so that

$$
\begin{aligned}
I_{1}(t, \varepsilon) & =\varepsilon^{2} \sqrt{\frac{t}{\varepsilon^{2}}} F\left(\frac{t}{\varepsilon^{2}}\right)=\varepsilon \sqrt{t} F\left(\frac{t}{\varepsilon^{2}}\right) \\
& =\varepsilon \sqrt{4 \pi t}\left(1-\frac{1}{4}\left(\frac{t}{\varepsilon^{2}}\right)+\mathcal{O}\left(\left(\frac{t}{\varepsilon^{2}}\right)^{2}\right)\right) .
\end{aligned}
$$

Next, for any smooth, bounded function $h$ on the $\varepsilon$-disk, consider the integral

$$
I_{2}(t, \varepsilon, h)=\int_{r=0}^{\varepsilon} \int_{0}^{\pi} e^{-\frac{r^{2}}{2 t}(1-\cos \theta)} r^{2} h(r, \theta) d \theta 2 r d r .
$$


Replacing $r$ with $\varepsilon r$, we obtain

$$
I_{2}(t, \varepsilon, h)=\varepsilon^{4} \int_{r=0}^{1} \int_{0}^{\pi} e^{-\frac{\varepsilon^{2} r^{2}}{2 t}(1-\cos \theta)} r^{2} h(\varepsilon r, \theta) d \theta 2 r d r .
$$

As before, we let $s=\frac{t}{\varepsilon^{2}}$, and we substitute $\cos \theta=\frac{x-s}{x+s}=1-\frac{2 s}{x+s}$.

$$
\begin{aligned}
I_{2}(t, \varepsilon, h) & =\varepsilon^{4} \int_{r=0}^{1} \int_{0}^{\infty} e^{-\frac{r^{2}}{x+s}} r^{2} h(\varepsilon r, \theta) \frac{\sqrt{s} d x}{\sqrt{x}(x+s)} 2 r d r \\
& =\varepsilon^{4} \sqrt{s} \int_{0}^{\infty} \int_{r=0}^{1} e^{-\frac{r^{2}}{x+s}} h(\varepsilon r, \theta) 2 r^{3} d r \frac{d x}{\sqrt{x}(x+s)} \\
& =\varepsilon^{4} \sqrt{s} G(s, \varepsilon),
\end{aligned}
$$

where we have used Fubini's theorem and the fact that the integral converges absolutely. Again, using a Lebesgue convergence theorem argument, it can be shown that the integral $G(s, \varepsilon)$ is smooth in $s$ and can be differentiated under the integral sign. Moreover, for any positive integer $m$,

$$
G(s, \varepsilon)=c_{0}(\varepsilon)+c_{1}(\varepsilon) s+c_{2}(\varepsilon) s^{2}+\ldots+c_{m}(\varepsilon) s^{m}+\mathcal{O}\left(s^{m+1}\right),
$$

where each $c_{j}(\varepsilon)$ remains bounded as $\varepsilon \rightarrow 0$. Therefore,

$$
\begin{aligned}
I_{2}(t, \varepsilon, h) & =\varepsilon^{4} \sqrt{\frac{t}{\varepsilon^{2}}} G\left(\frac{t}{\varepsilon^{2}}, \varepsilon\right)=\varepsilon^{3} \sqrt{t} G\left(\frac{t}{\varepsilon^{2}}, \varepsilon\right) \\
& =\varepsilon^{3} \sqrt{4 \pi t}\left(\bar{c}_{0}(\varepsilon)+\bar{c}_{1}(\varepsilon) \frac{t}{\varepsilon^{2}}+\mathcal{O}\left(\left(\frac{t}{\varepsilon^{2}}\right)^{2}\right)\right),
\end{aligned}
$$

where $\bar{c}_{0}(\varepsilon)$ and $\bar{c}_{1}(\varepsilon)$ are bounded as $\varepsilon \rightarrow 0$.

We will now compute the asymptotics of the integral over $D_{1}$ in equation (3.13). We have that

$$
\begin{aligned}
& \int_{D_{1}} \int_{-\pi}^{\pi} \widetilde{K}(t, w, w M(\theta)) \frac{d \theta}{2 \pi} \operatorname{dvol}_{W}(w) \\
= & \frac{1}{4 \pi t} \int_{r=0}^{\varepsilon} \int_{0}^{\pi} e^{-\frac{r^{2}}{2 t}(1-\cos \theta)}\left(b_{0}(r, \theta)+b_{1}(r, \theta) t+\mathcal{O}\left(t^{2}\right)\right) d \theta 2 r d r \\
= & \frac{1}{4 \pi t} \int_{r=0}^{\varepsilon} \int_{0}^{\pi} e^{-\frac{r^{2}}{2 t}(1-\cos \theta)}\left(b_{0}+\mathcal{O}\left(r^{2}\right)+b_{1} t+\mathcal{O}\left(r^{2} t\right)+\mathcal{O}\left(t^{2}\right)\right) d \theta 2 r d r \\
= & \frac{1}{4 \pi t}\left(I_{1}(t, \varepsilon)\left(b_{0}+b_{1} t\right)+I_{2}\left(t, \varepsilon, h_{0}\right)+I_{2}\left(t, \varepsilon, h_{1}\right) t+\mathcal{O}\left(I_{2}\left(t, \varepsilon, h_{2}\right) t^{2}\right)\right)
\end{aligned}
$$

for some appropriate choices of smooth, bounded functions $h_{0}, h_{1}$, and $h_{2}$. Note that we have denoted $b_{j}=\left.b_{j}(r, \theta)\right|_{r=0}$. Substituting the expressions (3.14) and (3.15), we get

$$
\begin{aligned}
& \int_{D_{1}} \int_{-\pi}^{\pi} \tilde{K}(t, w, w M(\theta)) \frac{d \theta}{2 \pi} \operatorname{dvol}_{W}(w) \\
= & \frac{1}{\sqrt{4 \pi t}}\left(\left(\varepsilon b_{0}+\mathcal{O}\left(\varepsilon^{3}\right)\right)+\left(-\frac{b_{0}}{4 \varepsilon}+\varepsilon b_{1}\right.\right. \\
& \left.+\mathcal{O}(\varepsilon)) t+\mathcal{O}\left(t^{2}\right)\right) .
\end{aligned}
$$


We observe that by the above construction, starting with the asymptotic expansion of $\widetilde{K}(t,(r, 0),(r, 0) M(\theta))$, we have $b_{0}=1$. This implies that

$$
\begin{aligned}
& \int_{D_{1}} \int_{-\pi}^{\pi} \widetilde{K}(t, w, w M(\theta)) \frac{d \theta}{2 \pi} \operatorname{dvol}_{W}(w) \\
= & \frac{1}{\sqrt{4 \pi t}}\left(O(\varepsilon)+\left(-\frac{1}{4 \varepsilon}+\mathcal{O}(\varepsilon)\right) t+\mathcal{O}\left(t^{2}\right)\right) .
\end{aligned}
$$

We have a similar formula for the asymptotics of the integral over the other $\varepsilon$-disk $D_{2}$. Thus, the integral over $W_{1}$ satisfies

$$
\begin{aligned}
& \int_{W_{1}} \int_{-\pi}^{\pi} \widetilde{K}(t, w, w M(\theta)) \frac{d \theta}{2 \pi} \operatorname{dvol}_{W}(w) \\
= & \frac{1}{\sqrt{4 \pi t}}\left(O(\varepsilon)+\left(-\frac{1}{2 \varepsilon}+\mathcal{O}(\varepsilon)\right) t+\mathcal{O}\left(t^{2}\right)\right) .
\end{aligned}
$$

A simple calculation shows that the $-\frac{1}{2 \varepsilon} t$ term exactly counteracts the blowing up of the term $a_{1}(w) t$ in (3.13) as $\varepsilon \rightarrow 0$, as expected. We remark that the analysis in this section could be extended in order to find the coefficients of larger powers of $t$ in the obvious way. Putting these results together, we have the following theorem.

Theorem 3.16. Suppose that $(M, \mathcal{F})$ is a Riemannian foliation of codimension two, and suppose that the leaf closures of $(M, \mathcal{F})$ have minimum codimension one, but some leaf closures have codimension two. Then the basic manifold $W$ is a sphere, and the $S O(2)$-action has exactly two fixed points $w_{1}$ and $w_{2}$. Let $W_{\varepsilon}=\left\{w \in W \mid \operatorname{dist}\left(w, w_{j}\right)>\varepsilon\right.$ for $\left.j=1,2\right\}$. As $t \rightarrow 0$, the trace $K_{B}(t)$ of the basic heat kernel on functions satisfies the following asymptotic expansion for any positive integer $J$ :

$$
K_{B}(t)=\frac{1}{\sqrt{4 \pi t}}\left(A_{0}+A_{1} t+A_{2} t^{2}+\ldots+A_{J} t^{J}+\mathcal{O}\left(t^{J+1}\right)\right),
$$

where

$$
A_{0}=\int_{M} \frac{1}{\operatorname{Vol}\left(\overline{L_{x}}\right)} \operatorname{dvol}(x)
$$

and in general $A_{j}=\lim _{\varepsilon \rightarrow 0}\left(\int_{W_{\varepsilon}} \bar{a}_{j}(w) \operatorname{dvol}(w)-f_{j}\left(\frac{1}{\varepsilon}\right)\right)$ for a specific polynomial $f_{j}$, and where $\bar{a}_{j}(w)=\phi(w) a_{j}\left(\pi\left(\rho^{-1}(w)\right)\right)$ with $a_{j}$ as in Theorem 3.15. As before, $\overline{L_{x}}$ denotes the leaf closure containing $x \in M$. Specifically,

$$
A_{1}=\lim _{\varepsilon \rightarrow 0}\left(\int_{W_{\varepsilon}} \frac{1}{\operatorname{Vol}\left(X_{w}\right)} S(w) \operatorname{dvol}(w)-\frac{1}{2 \varepsilon}\right)
$$

for

$$
S(w)=\frac{1}{2} K(w)+\frac{1}{4} \kappa(w)^{2}+\frac{\Delta_{W} \phi}{2 \phi}(w)+\frac{(d \phi, d \phi)}{4 \phi^{2}}(w),
$$

where $\phi(w)$ is the volume of the leaf closure $\rho^{-1}(w)$ in $\widehat{M}, X_{w}$ is the orbit of $w$ in $W, K(w)$ is the Gauss curvature of the basic manifold at $w$, and $\kappa(w)$ is the geodesic curvature of the orbit at $w$. 


\section{Examples}

In this section we compute two specific examples that demonstrate the behavior described in the last section. The first example is a transversally oriented, codimension two Riemannian foliation in which some of the leaf closures have codimension one and others have codimension two.

Example 4.1. Consider the one-dimensional foliation obtained by suspending an irrational rotation on the standard unit sphere $S^{2}$. On $S^{2}$ we use the cylindrical coordinates $(z, \theta)$, related to the standard rectangular coordinates by $x^{\prime}=$ $\sqrt{\left(1-z^{2}\right)} \cos \theta, y^{\prime}=\sqrt{\left(1-z^{2}\right)} \sin \theta, z^{\prime}=z$. Let $\alpha$ be an irrational multiple of $2 \pi$, and let the three-manifold $M=S^{2} \times[0,1] / \sim$, where $(z, \theta, 0) \sim(z, \theta+\alpha, 1)$. Endow $M$ with the product metric on $T_{z, \theta, t} M \cong T_{z, \theta} S^{2} \times T_{t} \mathbb{R}$. Let the foliation $\mathcal{F}$ be defined by the immersed submanifolds $L_{z, \theta}=\bigcup_{n \in \mathbb{Z}}\{z\} \times\{\theta+\alpha\} \times[0,1]$ (not unique in $\theta$ ). The leaf closures $\bar{L}_{z}$ for $|z|<1$ are two-dimensional, and the closures corresponding to the poles $(z= \pm 1)$ are one-dimensional. Therefore, this foliation satisfies the hypothesis of Theorem 3.16 .

In [47, we used this example to demonstrate the asymptotic behavior of $K_{B}(t, z, z)$ for different values of $z$. We state the results of some of the computations in 47. The basic functions are functions of $z$ alone, and the basic Laplacian on functions is $\Delta_{B}=-\left(1-z^{2}\right) \partial_{z}^{2}+2 z \partial_{z}$. The volume form on $M$ is $d z d \theta d t$, and the volume of the leaf closure at $z$ is $\frac{1}{2 \pi \sqrt{1-z^{2}}}$ for $|z|<1$. The eigenfunctions are the Legendre polynomials $P_{n}(z)$ corresponding to eigenvalues $m(m+1)$ for $m \geq 0$. From this information alone, one may calculate that the trace $K_{B}(t)$ of the basic heat operator is

$$
K_{B}(t)=\sum_{m \geq 0} e^{-m(m+1) t}=\frac{1}{\sqrt{4 \pi t}}\left(\pi+\frac{\pi}{4} t+\mathcal{O}\left(t^{2}\right)\right)
$$

The basic manifold $W$ corresponding to this foliation is a sphere with points described by orthogonal coordinates $(z, \varphi) \in[-1,1] \times(-\pi, \pi]$. As shown in [4], the metric on $W$ is given by $\left\langle\partial_{z}, \partial_{z}\right\rangle=\frac{1}{1-z^{2}},\left\langle\partial_{\varphi}, \partial_{\varphi}\right\rangle=\frac{4 \pi^{2}\left(1-z^{2}\right)}{4 \pi^{2}\left(1-z^{2}\right)+z^{2}}$. The following geometric quantities can be calculated from this data (see [47]):

$$
\begin{aligned}
\operatorname{dvol}_{z, \varphi} & =\frac{2 \pi}{\sqrt{4 \pi^{2}\left(1-z^{2}\right)+z^{2}}} d z d \varphi, \\
\operatorname{Vol}\left(X_{z}\right) & =\frac{4 \pi^{2} \sqrt{\left(1-z^{2}\right)}}{\sqrt{4 \pi^{2}\left(1-z^{2}\right)+z^{2}}}, \\
K(z, \varphi) & =2 \frac{\left(4 \pi^{2}-1\right) z^{2}+2 \pi^{2}}{\left(4 \pi^{2}\left(1-z^{2}\right)+z^{2}\right)^{2}}, \\
\frac{\Delta_{W} \phi}{2 \phi}(z, \varphi)+\frac{(d \phi, d \phi)}{4 \phi^{2}}(z, \varphi) & =\frac{\left(4 \pi^{2}-1\right)\left(\left(-3 \pi^{2}-\frac{3}{4}\right) z^{2}+\left(\pi^{2}-\frac{1}{4}\right) z^{4}+2 \pi^{2}\right)}{\left(4 \pi^{2}\left(1-z^{2}\right)+z^{2}\right)^{2}} \\
\kappa(z, \varphi)^{2} & =\frac{z^{2}}{\left(1-z^{2}\right)\left(4 \pi^{2}\left(1-z^{2}\right)+z^{2}\right)^{2}}, \\
\varepsilon & =\operatorname{dist~}\left(z=1, z=1-\varepsilon^{\prime}\right)=\frac{\pi}{2}-\arcsin \left(1-\varepsilon^{\prime}\right) .
\end{aligned}
$$


Using these computations, we may now compute $A_{0}$ and $A_{1}$ in Theorem 3.16 .

$$
A_{0}=\int_{M} \frac{1}{\operatorname{Vol}\left(\overline{L_{z}}\right)} \operatorname{dvol}(z, \theta, t)=\int_{t=0}^{1} \int_{\theta=-\pi}^{\pi} \int_{z=-1}^{1} \frac{1}{2 \pi \sqrt{1-z^{2}}} d z d \theta d t=\pi .
$$

Next, we substitute the functions into the formula for $A_{1}$ and simplify

$$
\begin{aligned}
A_{1} & =\lim _{\varepsilon \rightarrow 0}\left(\int_{W_{\varepsilon}} \frac{1}{\operatorname{Vol}\left(X_{w}\right)} S(w) \operatorname{dvol}(w)-\frac{1}{2 \varepsilon}\right) \\
& =\lim _{\varepsilon^{\prime} \rightarrow 0}\left(\int_{\varphi=-\pi}^{\pi} \int_{z=-1+\varepsilon^{\prime}}^{1-\varepsilon^{\prime}} \frac{1}{8 \pi} \frac{2-z^{2}}{\left(1-z^{2}\right)^{3 / 2}} d z d \varphi-\frac{1}{2\left(\frac{\pi}{2}-\arcsin \left(1-\varepsilon^{\prime}\right)\right)}\right) \\
& =\lim _{\varepsilon^{\prime} \rightarrow 0}\left(\int_{z=-1+\varepsilon^{\prime}}^{1-\varepsilon^{\prime}} \frac{1}{4} \frac{2-z^{2}}{\left(1-z^{2}\right)^{3 / 2}} d z-\frac{1}{2\left(\frac{\pi}{2}-\arcsin \left(1-\varepsilon^{\prime}\right)\right)}\right) \\
& =\lim _{\varepsilon^{\prime} \rightarrow 0}\left(\left.\frac{1}{4}\left(\frac{z}{\sqrt{\left(1-z^{2}\right)}}+\arcsin z\right)\right|_{-1+\varepsilon^{\prime}} ^{1-\varepsilon^{\prime}}-\frac{1}{2\left(\frac{\pi}{2}-\arcsin \left(1-\varepsilon^{\prime}\right)\right)}\right) \\
& =\lim _{\varepsilon^{\prime} \rightarrow 0}\left(\frac { 1 } { 2 } \left(\frac{1-\varepsilon^{\prime}}{\sqrt{\left(1-\left(1-\varepsilon^{\prime}\right)^{2}\right.}}+\arcsin \left(1-\varepsilon^{\prime}\right)\right.\right. \\
& \left.=-\frac{1}{2\left(\frac{\pi}{2}-\arcsin \left(1-\varepsilon^{\prime}\right)\right)}\right) \\
& \lim _{\varepsilon^{\prime} \rightarrow 0}\left(\frac{\pi}{4}+\mathcal{O}\left(\sqrt{\varepsilon^{\prime}}\right)\right)=\frac{\pi}{4} .
\end{aligned}
$$

Therefore, Theorem 3.16 implies that

$$
K_{B}(t)=\frac{1}{\sqrt{4 \pi t}}\left(\pi+\frac{\pi}{4} t+\mathcal{O}\left(t^{2}\right)\right)
$$

which agrees with the direct calculation (4.1).

The next example is an example of a codimension two, transversally oriented Riemannian foliation such that not all of the leaf closures are transversally orientable.

Example 4.2. This foliation is the suspension of an irrational rotation of the flat torus and a $\mathbb{Z}_{2}$-action. Let $X$ be any closed Riemannian manifold such that $\pi_{1}(X)=\mathbb{Z} * \mathbb{Z}$ - the free group on two generators $\{\alpha, \beta\}$. We normalize the volume of $X$ to be 1. Let $\widetilde{X}$ be the universal cover. We define $M=\widetilde{X} \times S^{1} \times S^{1} / \pi_{1}(X)$, where $\pi_{1}(X)$ acts by deck transformations on $\widetilde{X}$ and by $\alpha(\theta, \phi)=(2 \pi-\theta, 2 \pi-\phi)$ and $\beta(\theta, \phi)=(\theta, \phi+\sqrt{2} \pi)$ on $S^{1} \times S^{1}$. We use the standard product-type metric. The leaves of $\mathcal{F}$ are defined to be sets of the form $\left\{(x, \theta, \phi)_{\sim} \mid x \in \widetilde{X}\right\}$. Note that the foliation is transversally oriented, but the codimension one leaf closure foliation is not transversally orientable. The leaf closures are sets of the form

$$
\bar{L}_{\theta}=\left\{(x, \theta, \phi)_{\sim} \mid x \in \widetilde{X}, \phi \in[0,2 \pi]\right\} \cup\left\{(x, 2 \pi-\theta, \phi)_{\sim} \mid x \in \widetilde{X}, \phi \in[0,2 \pi]\right\} .
$$


The basic functions and one-forms are

$$
\begin{aligned}
& \Omega_{B}^{0}=\{f(\theta)\}, \\
& \Omega_{B}^{1}=\left\{g_{1}(\theta) d \theta+g_{2}(\theta) d \phi\right\},
\end{aligned}
$$

where the functions are smooth and satisfy

$$
\begin{aligned}
f(2 \pi-\theta) & =f(\theta), \\
g_{i}(2 \pi-\theta) & =-g_{i}(\theta) .
\end{aligned}
$$

From this information, we calculate that $\Delta_{B} f(\theta)=-f^{\prime \prime}(\theta)$. The eigenvalues $\{n \mid n \geq 0\}$ correspond to the eigenfunctions $\{\cos n \theta \mid n \geq 0\}$. Then

$$
\begin{aligned}
K_{B}(t, \theta, \theta) & =\frac{1}{2 \pi}+\frac{1}{\pi} \sum_{n \geq 1} e^{-n^{2} t} \cos ^{2}(n \theta) \\
& =\frac{1}{2 \pi} \sum_{n \in \mathbb{Z}} e^{-n^{2} t} \cos ^{2}(n \theta) \\
& =\frac{1}{4 \pi} \sum_{n \in \mathbb{Z}} e^{-n^{2} t}+\frac{1}{4 \pi} \sum_{n \in \mathbb{Z}} e^{-n^{2} t} \cos (2 n \theta) \\
& =\frac{1}{4 \pi} \operatorname{tr}\left(e^{-t \Delta} \text { on } L^{2}\left(S^{1}\right)\right)+\frac{1}{2} K(t, 0,2 \theta),
\end{aligned}
$$

where $K\left(t, \theta_{1}, \theta_{2}\right)$ is the heat kernel on functions on $S^{1}$. Substituting the expressions for this known kernel and its trace, we obtain

$$
\begin{aligned}
& K_{B}(t, \theta, \theta)=\frac{1}{4 \pi} \sqrt{\frac{\pi}{t}} \sum_{k \in \mathbb{Z}} e^{-k^{2} \pi^{2} / t}+\frac{1}{2} \frac{1}{\sqrt{4 \pi t}} \sum_{k \in \mathbb{Z}} e^{-(2 \theta+2 k \pi)^{2} / 4 t} \\
& \quad= \begin{cases}\frac{1}{2 \sqrt{\pi t}}+\mathcal{O}\left(e^{-\pi^{2} / t}\right) & \text { if } \theta=k \pi \text { for some } k \in \mathbb{Z}, \\
\frac{1}{4 \sqrt{\pi t}}+\mathcal{O}\left(e^{-c(\theta) / t}\right) & \text { otherwise, for } c(\theta)=\min \left\{(\theta+k \pi)^{2} \mid k \in \mathbb{Z}\right\} .\end{cases}
\end{aligned}
$$

The basic manifold $\widehat{W}$ is an $S O(2)$-manifold, defined by $\widehat{W}=[0, \pi] \times S^{1} / \sim$, where the circle has length 1 and $(\theta=0$ or $\pi, \gamma) \sim(\theta=0$ or $\pi,-\gamma)$. This is a Klein bottle, since it is the connected sum of two projective planes. The group $S O(2)$ acts on $\widehat{W}$ via the usual action on $S^{1}$. It is a simple exercise to calculate the trace of the basic heat operator from the eigenvalues alone:

$$
K_{B}(t)=\operatorname{tr}\left(e^{-t \Delta_{B}^{0}}\right)=\sum_{n \geq 0} e^{-n^{2} t} \sim \frac{\sqrt{\pi}}{2} t^{-1 / 2}+\frac{1}{2} .
$$

Note that the existence of the constant term above implies that the asymptotics of $K_{B}(t)$ cannot be obtained by integrating the asymptotics of $K_{B}(t, \theta, \theta)$ above.

We now compute the asymptotics of the trace of the basic heat operator using Theorem 3.10. The volume of a generic leaf closure is $4 \pi$, so the transverse volume is $A_{0}=V_{t r}=\frac{\operatorname{Vol}(M)}{4 \pi}=\pi$. The mean curvature of the leaf closures is identically zero, so that $A_{1}=B_{1}=0$. Thus, Theorem 3.10 implies that

$$
K_{B}(t)=\frac{1}{\sqrt{4 \pi t}}\left(\pi+\sqrt{\pi} t^{1 / 2}+\mathcal{O}\left(t^{2}\right)\right) \sim \frac{\sqrt{\pi}}{2} t^{-1 / 2}+\frac{1}{2},
$$

as expected. 


\section{Riemannian fOliations that ARE nOt TRANSVERSALly ORIENTABLE}

In most of the cases considered throughout this paper, we have assumed that the foliation in question is transversally oriented. We now remark that with a few simple modifications, the results of this paper can be used to find the asymptotics of the trace of the basic heat operator on a Riemannian foliation that is not transversally orientable. First of all, the basic Laplacian on functions is still well-defined on such foliations; the basic Laplacian can be defined using a local orientation and does not depend on the choice of that orientation. Suppose that $(M, \mathcal{F})$ is a Riemannian foliation on a connected, compact manifold with a bundle-like metric such that the leaves are not transversally orientable. The foliation may now be lifted to the (nonoriented) orthonormal transverse frame bundle, an $O(q)$ bundle over $M$. The lifted foliation is transversally parallelizable, and the closures of the leaves of the lifted foliation fiber over a compact $O(q)$-manifold $\bar{W}$. The group $O(q)$ does not act by orientation-preserving isometries, but otherwise the results of this paper extend by letting the group $G=O(q)$. Thus, Theorem 2.3 holds, but some of the results in Section 3 would have to be modified to allow for orientation-reversing holonomy. For example, the powers of $t$ in the asymptotic expansions of the trace of the basic heat operator would in general increment by half integers instead of integers. Note that this phenomenon can occur even in the transversally oriented case, if the leaf closures are not necessarily transversally oriented (see Example 4.2).

\section{ACKNOWLEDGEMENT}

The author thanks George Gilbert, Efton Park, Franz Kamber, and Jochen Brüning for helpful conversations.

\section{REFERENCES}

[1] J. A. Álvarez López, The basic component of the mean curvature of Riemannian foliations, Ann. Global Anal. Geom. 10(1992), 179-194. MR.1175918 (93h:53027)

[2] M. F. Atiyah, R. Bott, and V. K. Patodi, On the heat equation and index theorem, Invent. Math. 19(1973), 279-330. MR0650828 (58:31287)

[3] M. Berger, Sur les spectre d'une variété riemannienne, C. R. Acad. Sci. Paris Sér. I Math. 163(1963), 13-16. MR0158332 (28:1557)

[4] M. Berger, P. Gauduchon, and E. Mazet, Le Spectre d'Une Variété Riemannienne, Springer Verlag, Berlin, 1971. MR0282313 (43:8025)

[5] N. Berline, E. Getzler, and M. Vergne, Heat Kernels and Dirac Operators, Springer Verlag, Berlin, 1991. MR,1215720 (94e:58130)

[6] D. Bleecker, The supertrace of the steady asymptotic of the spinorial heat kernel, J. Math. Phys. 33(1992), no. 6, 2053-2070. MR1164316 (93d:58153)

[7] G. Bredon, Introduction to Compact Transformation Groups, Academic Press, New York, 1972. MR0413144 (54:1265)

[8] T. Bröcker and T. tom Dieck, Representations of Compact Lie Groups, Springer-Verlag, New York, 1985. MR781344 (86i:22023)

[9] J. Brüning and E. Heintze, Representations of compact Lie groups and elliptic operators, Inventiones Math. 50(1979), 169-203. MR.517776 (81b:58039)

[10] J. Brüning and E. Heintze, The asymptotic expansion of Minakshisundaram-Pleijel in the equivariant case, Duke Math. J. 51(1984), 959-979. MR771390(86b:58124)

[11] J. Brüning and F. W. Kamber, Vanishing theorems and index formulas for transversal Dirac operators, A.M.S. Meeting 845, Special Session on Operator Theory and Applications to Geometry, Lawrence, Kansas, A.M.S. Abstracts, October 1988.

[12] R. Camporesi, The spinor heat kernel in maximally symmetric spaces, Comm. Math. Phys. 148(1992), no. 2, 283-308. MR1178146 (93g:58140) 
[13] R. A. Carmona and W. A. Zheng, Reflecting Brownian motions and comparison theorems for Neumann heat kernels, J. Funct. Anal. 123(1994), no. 1, 109-128. MR.1279298 (95k:60202)

[14] I. Chavel, Eigenvalues in Riemannian geometry, Academic Press, New York, 1984. MR768584 (86g:58140)

[15] J. Cheeger, Analytic torsion and the heat equation, Ann. of Math. 109(1979), 259-322. MR528965 (80j:58065a)

[16] M. Craioveanu and M. Puta, Asymptotic properties of eigenvalues of the basic Laplacian associated to certain Riemannian foliations, Bull. Math. Soc. Sci. Math. Roumanie (N.S.) 35(83)(1991), no. 1-2 , 61-65. MR.1307911 (95j:58164)

[17] H. Donnelly, Spectrum and the fixed point sets of isometries I, Math. Ann. 224(1976), 161170. MR0420743 (54:8755)

[18] H. Donnelly, Asymptotic expansions for the compact quotients of properly discontinuous group actions, Illinois J. Math. 23(1979), 485-496. MR.537804 (80h:58049)

[19] E. B. Dryden, C. S. Gordon, S. J. Greenwald, and D. L. Webb, Asymptotic expansion of the heat kernel for orbifolds, Mich. Math. J., 56 (2008), no. 1, 205-238. MR2433665 (2009h:58057)

[20] A. El Kacimi-Alaoui, Équation de la chaleur sur les espaces singulièrs, C. R. Acad. Sci. Paris 303(1986) no. 6, 243-246. MR860827 (87k:58258)

[21] A. El Kacimi-Alaoui and G. Hector, Décomposition de Hodge basique pour un feuilletage Riemannien, Ann. Inst. Fourier, Grenoble 36(1986), no. 3, 207-227. MR865667|(87m:57029)

[22] G. Esposito, G. Fucci, A. Y. Kamenshchik, and K. Kirsten, Spectral asymptotics of Euclidean quantum gravity with diff-invariant boundary conditions, Classical Quantum Gravity 22(2005), no. 6, 957-974. MR2131582 (2005m:83044)

[23] H. D. Fegan, The heat equation and modular forms, J. Diff. Geo. 13(1978), 589-602. MR570220 (81k:22006)

[24] W. Feller, An Introduction to Probability Theory and Its Applications, Vol. II, Wiley, New York, 1966. MR0210154 (35:1048)

[25] P. B. Gilkey, Invariance theory, the heat equation, and the Atiyah-Singer Index Theorem, Publish or Perish, Wilmington, DE, 1984. MR783634 (86j:58144)

[26] J. F. Glazebrook and F. W. Kamber, Transversal Dirac families in Riemannian foliations, Comm. Math. Phys. 140(1991), 217-240. MR1124268 (92j:58103)

[27] P. Greiner, An asymptotic expansion for the heat equation, Arch. Rational Mech. Anal. 41(1971), 163-218. MR0331441(48:9774)

[28] F. B. Hildebrand, Advanced Calculus for Applications, second ed., Prentice Hall, Inc., Englewood Cliffs, N.J., 1976.

[29] F. W. Kamber and Ph. Tondeur, De Rham-Hodge theory for Riemannian foliations, Math. Ann. 277(1987), 415-431. MR891583 (89d:53070)

[30] K. Kawakubo, The Theory of Transformation Groups, Oxford University Press, 1991. MR.1150492 (93g:57044)

[31] J. Lee and K. Richardson, Riemannian foliations and eigenvalue comparison, Ann. Global Anal. Geom. 16(1998), no. 6, 497-525. MR.1651376 (99k:53047)

[32] P. Malliavin and D. Stroock, Short time behavior of the heat kernel and its logarithmic derivatives, J. Diff. Geom. 44(1996), 550-570. MR.1431005 (98c:58164)

[33] H. P. McKean and I. M. Singer, Curvature and the eigenvalues of the Laplacian, J. Diff. Geom. 1(1967), 43-69. MR0217739 (36:828)

[34] J. Milnor, Morse Theory, Ann. Math. Stud., no. 51, Princeton University Press, 1963. MR0163331 (29:634)

[35] S. Minakshisundaram and A. Pleijel, Some properties of the eigenfunctions of the Laplaceoperator on Riemannian manifolds, Canadian J. Math. 1(1949), 242-256. MR0031145 $(11: 108 \mathrm{~b})$

[36] P. Molino, Riemannian foliations, Progress in Mathematics, Birkhauser, Boston, 1988. MR.932463 (89b:53054)

[37] S. Nishikawa, M. Ramachandran, and Ph. Tondeur, The heat equation for Riemannian foliations, Trans. Amer. Math. Soc. 319(1990), 619-630. MR987165 (90j:58145)

[38] S. Nishikawa, Ph. Tondeur, and L. Vanhecke, Spectral geometry for Riemannian foliations, Ann. Global Anal. Geom. 10(1992), 291-304. MR1186017 (93h:58160)

[39] B. Osgood, R. Phillips, and P. Sarnak, Extremals of Determinants of Laplacians, J. Funct. Anal. 80(1988), no. 1, 148-211. MR960228 (90d:58159) 
[40] B. Osgood, R. Phillips, and P. Sarnak, Compact Isospectral Sets of Surfaces, J. Funct. Anal. 80(1988), no. 1, 212-234. MR960229 (90d:58160)

[41] E. Park, Toeplitz algebras associated to isometric flows, Illinois J. Math. 41(1997), no. 1, 93-102. MR1433188 (98g:47023)

[42] E. Park and K. Richardson, The basic Laplacian of a Riemannian foliation, Amer. J. Math. 118(1996), 1249-1275. MR1420923 (97i:58165)

[43] A. S. Petrow, Einstein-Räume, Akademie-Verlag, Berlin, 1964. MR0162594 (28:5792)

[44] D.B. Ray and I.M. Singer, R-Torsion and the Laplacian on Riemannian manifolds, Adv. Math. 7(1971), 145-210. MR0295381 (45:4447)

[45] B. Reinhart, Differential Geometry of Foliations, Springer-Verlag, Berlin, 1983. MR.705126 (85i:53038)

[46] K. Richardson, Critical points of the determinant of the Laplace operator, J. Funct. Anal. 122(1994), no. 1, 52-83. MR1274583 (95j:58176)

[47] K. Richardson, The asymptotics of heat kernels on Riemannian foliations, Geom. Funct. Anal. 8(1998), 1-46. MR.1616151 (99e:58188)

[48] K. Richardson, The transverse geometry of G-manifolds and Riemannian foliations, Illinois J. Math. 45(2001), 517-535. MR1878616 (2002k:53041)

[49] J. Roe, Elliptic operators, topology, and asymptotic methods, Pitman Research Notes in Math., no. 179, Longman Scientific and Technical, Harlow, and Wiley, New York, 1988. MR960889 (89j:58126)

[50] Ph. Tondeur, Foliations on Riemannian manifolds, Springer Verlag, New York, 1988. MR.934020 (89e:53052)

[51] H. Weyl, Der Asymptotische Verteilungsgesetz der Eigenwerte linearer partieller Differentialgleichungen, Math. Ann. 71(1912), 441-469. MR1511670

[52] H. E. Winkelnkemper, The graph of a foliation, Ann. Global Anal. Geom. 1(1983), no. 3, 51-75. MR739904 (85j:57043)

Department of Mathematics, Texas Christian University, TCU Box 298900, Fort Worth, TEXas 76129

E-mail address: k.richardson@tcu.edu 